\title{
Simulating electron and ion temperature in a global ionosphere thermosphere model: validation and modeling an idealized substorm
}

\author{
Jie Zhu ${ }^{\mathrm{a}}$, Aaron J. Ridley ${ }^{\mathrm{a}}$, Yue Deng ${ }^{\mathrm{b}}$ \\ ${ }^{a}$ Department of Atmospheric, Oceanic and Space Sciences, University of Michigan, Ann Arbor, Michigan, USA. \\ ${ }^{b}$ Department of Physics, University of Texas, Arlington, USA.
}

\section{(C) 2011 Published by Elsevier Ltd.}

\section{Introduction}

\begin{abstract}
Electron and ion temperatures control many chemical and physical processes in the ionosphere-thermosphere system. Recently, improved electron and ion energy equations were implemented in the Global Ionosphere Thermosphere Model (GITM). The source energy of the electron temperature $\left(T_{e}\right)$ include thermal conduction, heating due to photoionization, elastic collisions with ions, elastic and inelastic collisions with neutrals, auroral precipitation, and heat flux from inner magnetosphere. The source terms in the ion temperature $\left(T_{i}\right)$ equation include thermal conduction, and elastic collisions with electrons and neutrals. The new implementation of $T_{e}$ improved the ionospheric density at middle and high latitudes with respect to IRI. The improved GITM also reproduced the diurnal variation in $T_{e}$ and $T_{i}$ observed by incoherent scatter radars at low and middle latitudes. The model was used to investigate an idealized substorm statistically described by Clausen et al. [1]. It was found that the responses of the E-region $N_{e}$ and $T_{e}$ were highly correlated with the variation in auroral hemispheric power. The change of the F-region $T_{e}$ was correlated with the E-region $T_{e}$ and $N_{e}$, which was consistent with observations. The response of the F-region $N_{e}$ to the particle precipitation was delayed by about 30 minutes, and lasted significantly longer than the enhanced precipitation. The variations of $T_{i}$ in both the Eand F-regions were dominated by IMF-driven ion drifts through frictional energy coupling with the neutrals. It was also found that the increase of the mid-latitude heat flux by one order of magnitude enhanced Te, electron density and TEC by up to $120 \%, 80 \%$ and $80 \%$ respectively between dusk and midnight.
\end{abstract}

Keywords: Electron Temperature, Ion Temperature, Ionosphere

Electron and ion temperatures strongly affect the ionosphere-thermosphere system because they control many chemical and physical processes [2]; therefore, investigating the temporal and spatial variations of the temperatures is important in understanding the coupled system. It has been widely observed that variations exist in the F-region $T_{e}$ at low and middle latitudes, which are characterized by a "morning overshoot" [3, 4] and an evening enhancement [5].

${ }^{*}$ Corresponding author. Fax: +1734 6473083

Email address: zhjie@umich.edu (Jie Zhu) 
These morning and evening enhancements are mainly caused by the photoelectron heating of the low-density thermal electrons at dawn and dusk $[6,7,8]$. The morning peak tends to occur in equinox and winter at high solar activity at Millstone Hill, while the evening enhancement is weaker or absent. At Arecibo, the morning enhancement occurs during all seasons, while the evening enhancement only tends to occur in the equinox and winter seasons [9]. Truhlik et al. [10] found an anti-correlation between the daytime $T_{e}$ and solar activity at mid-latitudes below $700 \mathrm{~km}$ during the equinox and winter, while the nighttime $T_{e}$ increased with solar activity at low and mid-latitudes at all altitudes. $T_{e}$ can also be significantly enhanced by heat flux from the plasmasphere in regions with low electron density, especially inside the midlatitude electron density trough [11]. The high-latitude $T_{e}$ is strongly affected by the polar heat flux and field-aligned current (FAC) [12]. A downward polar heat flux can affect the electron density and temperature near the F-region and above [13]. Upward (Downward) FACs increase (decrease) $T_{e}$ at altitudes higher than the F-region maximum [14].

Banks [15] suggested a transition behavior of $T_{i}$, which increased from the neutral temperature near $250 \mathrm{~km}$ towards $T_{e}$ at higher altitudes. The daytime high-latitude $T_{i}$ generally tends to be higher in summer than in winter, at solar maximum than at solar minimum, and for active magnetic conditions than for quiet conditions [16]. They also found that an upward heat flow from the lower ionosphere due to the meridional electric fields acts to raise $T_{i}$ at high altitudes. The high-latitude F-region $T_{i}$ tends to be enhanced by ion-neutral friction heating during geomagnetic storms, leading to an increase in $T_{e}$ due to the ion-electron energy transfer [11]. $T_{i}$ is strongly related to the ion-neutral collision frequencies that affect the heat transfer rates between ions and neutrals. The ion energy equation has been approximated by a balance between the energy exchange and frictional heating with neutrals below $400 \mathrm{~km}$ at high latitudes [17].

Efforts have been made in modeling $T_{e}$ and $T_{i}$ in the ionosphere since the 1980s. The Time-Dependent Ionosphere Model (TDIM) solves the electron energy equation considering thermal conduction, thermoelectric transport, ion Joule heating, heating due to photoelectrons and auroral electrons, and collisions with the thermal ions and neutrals $[12,18,19,13]$. The Coupled Thermosphere-Ionosphere-Plasmasphere (CTIP) model solves the time-dependent $T_{e}$ and $T_{i}$ for $\mathrm{O}^{+}$and $\mathrm{H}^{+}$in field-aligned coordinates. The sources terms include heating due to collisional interactions with other species, adiabatic heating/cooling and thermal conductivity [20]. SAMI2 solves similar ion and electron energy equations in field-aligned coordinates with a semi-implicit method [21]. $T_{e}$ in the Thermosphere-Ionosphere Nested Grid (TING) model and the Thermosphere Ionosphere Electrodynamics General Circulation Model (TIEGCM) is solved assuming a steady state with vertical heat conduction and heating due to photoelectrons and particle precipitation, as well as cooling to ions and neutrals $[22,11]$.

Since the early 1980s, radar and rocket observations have been used to study the ionospheric dynamics during 
substorms [23, 24, 25]. Maehlum et al. [26] found that the F-region electron temperature was correlated with the E-region density based on the EISCAT observations during an intense aurora. They suggested that the F-region could be heated by current driven instabilities associated with intense particle precipitation. Rocket observation showed that the ionospheric electron temperature was significantly higher than that would be due to collisional heating with precipitating particles and it was suggested that some wave-particle interaction contributed the extra heating [27]. The F-region $T_{i}$ measured by EISCAT UHF-radar was found to be dominated by the local electric field [28]. During the expansion phase of an isolated substorm, both $T_{e}$ and $N_{e}$ were strongly enhanced [29]. Although the thermal response of the ionosphere during a substorm has been greatly studied, there have been few studies using global ionosphere thermosphere model to investigate the response of the ionospheric temperatures during substorms. In this study, the Global Ionosphere Thermosphere Model (GITM) with the recently implemented ionospheric temperature model, was used to explore the response of the ionosphere to an idealized substorm $[1,30]$ and the effect of the topside electron heat flux.

\section{Model description}

The Global Ionosphere Thermosphere Model is a three-dimensional model that couples the ionosphere-thermosphere system in spherical coordinates [31]. GITM solves the continuity, momentum and energy equations with realistic source terms and a modern advection solver. GITM solves for the full chemical reactions for ion species: $O^{+}\left({ }^{4} S\right)$, $\mathrm{O}^{+}\left({ }^{2} \mathrm{D}\right), \mathrm{O}^{+}\left({ }^{2} \mathrm{P}\right), \mathrm{O}_{2}^{+}, \mathrm{N}^{+}, \mathrm{N}_{2}^{+}$and $\mathrm{NO}^{+}$. The computational domain spans from $\sim 95 \mathrm{~km}$ to $650 \mathrm{~km}$ in altitude. The ion momentum equation is solved in steady state with pressure gradient, gravity, neutral wind drag and external electric fields. $\mathrm{O}^{+}$is currently advected in GITM since it is the dominant ion in the low collision region of the ionosphere. The dynamo electric field is solved for in a self-consistent way by using the technique of Richmond [32], as described by Vichare et al. [33]. Different models of high-latitude potential and aurora precipitation are allowed in GITM. In this study, the Weimer [34] model was used for the high-latitude electric fields, and the Fuller-Rowell and Evans [35] model was employed for produce the auroral precipitation patterns.

\subsection{Electron Temperature}

Instead of assuming a steady state electron temperature, as was done in earlier version of GITM, the new model solves for a time-dependent electron energy equation. This allows a (possibly) more precise description of the temporal variations of $T_{e}$. If chemical reactions and viscous heating of the electron gas are neglected, the electron energy equation can be expressed as [36]: 


$$
\frac{3}{2} \kappa n_{e} \frac{\partial T_{e}}{\partial t}=-\kappa n_{e} T_{e} \nabla \cdot \boldsymbol{u}_{e}-\frac{3}{2} N_{e} \kappa \boldsymbol{u}_{e} \cdot \nabla T_{e}-\nabla \cdot \boldsymbol{q}_{e}+\Sigma Q_{e}
$$

where $\kappa$ is the Boltzmann constant, $n_{e}$ is the electron density, $T_{e}$ is the electron temperature, $\boldsymbol{u}_{\boldsymbol{e}}$ is the electron velocity, $\boldsymbol{q}_{\boldsymbol{e}}$ is the heat flow vector, $t$ is time and $z$ is altitude. The terms on the right-hand side of Eqn(1) represent (from left to right) the adiabatic expansion, heat advection, and the divergence of the electron heat flow vector respectively. $\Sigma Q_{e}$ is the sum of all the local heating and cooling rates, respectively. In this model, the adiabatic heating/cooling is neglected. The heat flow vector is expressed as

$$
\boldsymbol{q}_{e}=-\chi \boldsymbol{J}_{\|}-\lambda_{e} \nabla T_{e}
$$

where $\chi$ is the thermoelectric coefficient, $\chi=\frac{5}{2} \kappa T_{e} / e$ [36] and $\lambda_{e}$ is the electron thermal conductivity (given in the Appendix). This model also included the thermoelectric heating along the field lines above $45^{\circ}$ Magnetic Latitude. The field aligned current is based on the divergence of the ion and electron flows in the plane perpendicular to the field lines. Considering thermal advection only in the radial direction, the electron energy equation implemented in the new model can be simplified to:

$$
\frac{3}{2} \kappa n_{e} \frac{\partial T_{e}}{\partial t}=-\frac{3}{2} \kappa n_{e} u_{e} \frac{\partial T_{e}}{\partial r}+Q_{J_{/ /}}+Q_{\text {conduction }}+\Sigma Q_{e}
$$

where $r$ is the unit vector in the radial direction in the Earth's spherical coordinates. The terms on the right side represent (from left to right) the thermal advection in the radial direction, thermoelectric heating, thermal conduction and the sum of other local heating/cooling sources. The field aligned thermal conduction can be written as:

$$
Q_{\text {conduction }}=\frac{\partial}{\partial s}\left(\lambda_{e} \frac{\partial T_{e}}{\partial s}\right)=\lambda_{e} \frac{\partial^{2} T_{e}}{\partial s^{2}}+\frac{\partial \lambda_{e}}{\partial s} \frac{\partial T_{e}}{\partial s}
$$

Since the electron thermal conduction primarily occurs along magnetic field lines [12], there has been no global ionosphere thermosphere model that includes the thermal conduction perpendicular to field lines, to the authors' knowledge. In this model, thermal conduction both along and perpendicular to the field lines are implemented. Projecting the field-aligned conduction to spherical coordinates gives: 


$$
\begin{aligned}
& Q_{\text {conduction }}=\sin ^{2} \alpha\left(\lambda_{e} \frac{\partial^{2} T_{e}}{\partial r^{2}}+\frac{\partial T_{e}}{\partial r} \frac{\partial \lambda_{e}}{\partial r}\right) \\
& +\lambda_{e} \cos ^{2} \alpha\left(\frac{1}{r^{2}} \cos ^{2} \beta \frac{\partial^{2} T_{e}}{\partial \theta^{2}}+\frac{\sin ^{2} \beta}{r^{2} \cos ^{2} \theta} \frac{\partial^{2} T_{e}}{\partial \phi^{2}}+\frac{2 \sin \beta \cos \beta}{r^{2} \cos \theta} \frac{\partial^{2} T_{e}}{\partial \theta \partial \phi}-\frac{\sin \beta \cos \beta \sin \theta}{r^{2} \cos ^{2} \theta} \frac{\partial T_{e}}{\partial \phi}\right) \\
& +\cos ^{2} \alpha\left(\cos ^{2} \beta \frac{1}{r^{2}} \frac{\partial \lambda_{e}}{\partial \theta} \frac{\partial T_{e}}{\partial \theta}+\sin ^{2} \beta \frac{1}{r^{2} \cos ^{2} \theta} \frac{\partial \lambda_{e}}{\partial \phi} \frac{\partial T_{e}}{\partial \phi}+\sin \beta \cos \beta \frac{1}{r^{2} \cos \theta}\left(\frac{\partial \lambda_{e}}{\partial \theta} \frac{\partial T_{e}}{\partial \phi}+\frac{\partial \lambda_{e}}{\partial \phi} \frac{\partial T_{e}}{\partial \theta}\right)\right. \\
& +\sin \alpha \cos \alpha \cos \beta \frac{1}{r}\left(\frac{\partial T_{e}}{\partial \theta} \frac{\partial \lambda_{e}}{\partial r}+\frac{\partial T_{e}}{\partial r} \frac{\partial \lambda_{e}}{\partial \theta}\right)+\sin \alpha \cos \alpha \sin \beta \frac{1}{r \cos \theta}\left(\frac{\partial T_{e}}{\partial \phi} \frac{\partial \lambda_{e}}{\partial r}+\frac{\partial T_{e}}{\partial r} \frac{\partial \lambda_{e}}{\partial \phi}\right),
\end{aligned}
$$

where $\alpha$ and $\beta$ are the magnetic dip and declination angle, respectively. $\theta$ and $\phi$ are the latitude and longitude in the Earth's spherical coordinates, respectively. The cross terms between the radial direction and the horizontal plane, i.e., the last two terms in Eqn(5), were ignored for simplicity.

Considering the field-aligned currents are prominent in high latitudes, the heating/cooling due to field-aligned current as considered above $45^{\circ}$ Magnetic Latitude. The field aligned current $J_{/ /}$was calculated as:

$$
J_{/ /}=\int_{r_{0}}^{r_{1}} \nabla \cdot J_{\perp}
$$

where $r_{0}$ and $r_{1}$ are the lower and upper boundary of the ionosphere, respectively. $J_{\perp}$ is the current perpendicular to the field lines, and $J_{\perp}=n_{e}|e|\left(u_{i \perp}-u_{e \perp}\right)$. Assuming the field aligned current is mainly contributed by electron flow and using the relation $J_{/ /}=-n_{e}|e| u_{e}$, the thermal advection term as expressed as a function of $J_{/ /}$:

$$
-\frac{3}{2} \kappa n_{e} u_{e} \frac{\partial T_{e}}{\partial r}=\frac{3}{2} \kappa \frac{J_{/ /}}{|e|} \frac{\partial T_{e}}{\partial r},
$$

and was included above $45^{\circ}$ Magnetic Latitude.

The local heating/cooling rates are given by:

$$
\Sigma Q_{e}=Q_{\text {phe }}+Q_{\text {auro }}+Q_{e i}+\left[Q_{e n}\right]_{\text {elas }}+\left[Q_{\text {en }}\right]_{\text {inelas }},
$$

where $Q_{p h e}$ is heating due to photoelectrons, $Q_{a u r o}$ is heating due to auroral ionization, $Q_{e i}$ is the heat transfer between electrons and ions due to elastic collisions, and $\left[Q_{e n}\right]_{e l a s}$ and $\left[Q_{e n}\right]_{\text {inelas }}$ represent cooling rates due elastic and inelastic collisions with neutrals. The new model adds cooling due to elastic collisions with neutrals and heating due to auroral ionization, and calculates the photoelectron heating using an improved parameterization model of photoelectron heating efficiency described by Smithtro and Solomon [37], which allows for a change in the heating efficiency as a function of altitude due to the changing of the ratio between the electron and neutral densities. The photoelectron heating can be expressed as: 


$$
Q_{\text {phe }}=\epsilon P_{\text {photo }},
$$

where $\epsilon$ is a thermal electron heating efficiency and $P_{\text {photo }}$ is the local photoionization rate [38]. The heating efficiency is specified by an improved parameterization derived by Smithtro and Solomon [37]:

$$
\begin{aligned}
\epsilon=\exp & \left(5.342+1.056 x-4.392 \times 10^{-2} x^{2}-5.900 \times 10^{-2} x^{3}\right. \\
& \left.-9.346 \times 10^{-3} x^{4}-5.755 \times 10^{-4} x^{5}-1.249 \times 10^{-5} x^{6}\right),
\end{aligned}
$$

where

$$
x=\ln (R), \quad R=\frac{n_{e}}{n\left(N_{2}\right)+n\left(O_{2}\right)+n(O)} .
$$

This parameterization performs over a wide altitude range of $\sim 100 \mathrm{~km}$ to $\sim 750 \mathrm{~km}[39,40]$. The domain of GITM, from $\sim 90 \mathrm{~km}$ to $\sim 700 \mathrm{~km}$, is basically within its applicable range. The maximum of the ratio $R$ in Eqn(11) is limited to 10 in the model, which rarely, if ever, actually happens, even at high solar activity. The auroral heating rate is expressed as

$$
Q_{\text {auro }}=\epsilon P_{\text {auro }},
$$

where $P_{\text {auro }}$ is the auroral ionization rate, and the heating efficiency $\epsilon$ is the same as shown in Eqn(10).

The heat transfer due to elastic collisions with ions is given by:

$$
Q_{e i}=3 \kappa n_{e} m_{e} \sum_{t} v_{e t} \frac{\left(T_{i}-T_{e}\right)}{m_{e}+m_{t}}
$$

where $m_{e}$ is the electron mass, and $T_{i}$ is the ion temperature (note that GITM only has a single bulk ion temperature). The coulomb collision frequency $\left(v_{e t}\right)$ between electrons and ion species $t$ is given in Appendix A.

The heating term due to collisions with neutrals consists of two parts: elastic collisions and inelastic collisions. The elastic collision term is given by:

$$
\left[Q_{e n}\right]_{e l a s}=3 \kappa n_{e} m_{e} \sum_{s} v_{e s} \frac{T_{n}-T_{e}}{m_{e}+m_{s}},
$$

where $T_{n}$ is the neutral temperature and $v_{e s}$ is the collision frequency between electrons and neutral species $s$ and is given in Appendix A. This elastic collision term is almost always a cooling term, due to the fact that the neutrals are almost always cooler than the electrons. The corresponding heating rate for neutrals due to elastic collisions with 
electrons is a source term in the neutral energy equation.

The inelastic collision term is given by:

$$
\left[Q_{e n}\right]_{\text {inelas }}=L_{e}\left(N_{2}\right)_{\text {rot }}+L_{e}\left(O_{2}\right)_{\text {rot }}+L_{e}\left(N_{2}\right)_{\text {vib }}+L_{e}\left(O_{2}\right)_{\text {vib }}+L_{e}(O)_{\text {fine }}+L_{e}\left(O\left({ }^{1} D\right)\right),
$$

where $L_{e}\left(N_{2}\right)_{\text {rot }}$ and $L_{e}\left(O_{2}\right)_{\text {rot }}$ are the cooling rates due to the rotational excitation of the molecular neutrals $N_{2}$ and $O_{2}, L_{e}\left(N_{2}\right)_{v i b}$ and $L_{e}\left(O_{2}\right)_{v i b}$ are due to vibrational excitation of $N_{2}$ and $O_{2}, L_{e}(O)_{\text {fine }}$ and $L_{e}\left(O\left({ }^{1} D\right)\right)$ are due to the fine structure and the excitation of atomic oxygen to the $O\left({ }^{1} D\right)$ state (given in Appendix A).

\subsection{Ion Temperature}

The old $T_{i}$ calculation in GITM was based on a statistical formula assuming a balance between the neutral energy exchange term and the neutral frictional heating term [17]. In the new model, the physical ion energy equation including thermal diffusion and collisional heat transfer between the ions and the electrons and neutrals is implemented. All the ion species sharing the same grid volume are assumed to have the same temperature. The ion temperature equation can be expressed as:

$$
\frac{3}{2} \kappa n_{i} \frac{\partial T_{i}}{\partial t}=-\kappa n_{i} T_{i} \nabla \cdot \boldsymbol{u}_{i}-\frac{3}{2} N_{i} \kappa \boldsymbol{u}_{i} \cdot \nabla T_{i}-\nabla \cdot \boldsymbol{q}_{i}+\Sigma Q_{i}
$$

In GITM, the adiabatic expansion and heat advection were neglected. Also, considering field-aligned currents were mainly driven by electron motion, the thermoelectric heating was ignored in GITM. Thus, the heat flow vector $\boldsymbol{q}_{i}$ is:

$$
\boldsymbol{q}_{i}=-\lambda_{i} \nabla T_{i}
$$

where $\lambda_{i}$ is the ion thermal conductivity given in Appendix B.

Similar to electrons, the ion thermal conduction was considered along the magnetic field, both in the vertical and horizontal directions. With these assumptions, the ion energy equation can be written as:

$$
\frac{3}{2} \kappa n_{i} \frac{\partial T_{i}}{\partial t}=Q_{\text {conduction }}+\Sigma Q_{i}
$$

$$
\begin{aligned}
& \text { where } n_{i} \text { is the total ion density (equal to the electron density) and } T_{i} \text { is the ion temperature. The ion thermal con- } \\
& \text { duction is in the same format as the electron thermal conduction in Eqn (5). } Q_{i} \text { represents the total local ion heat- } \\
& \text { ing/cooling rate, which is the sum of the heat transfer due to collisions with electrons and with neutrals. }
\end{aligned}
$$

The elastic collisional heating of the ions by the electrons is: 


$$
Q_{i e}=3 \kappa \sum_{t} \frac{n_{t} m_{t} v_{t e}}{m_{t}+m_{e}}\left(T_{e}-T_{i}\right)
$$

where the subscript $t$ refers to the ion species. Given that the collision frequencies satisfy the relationship [2]:

$$
n_{t} m_{t} v_{t e}=n_{e} m_{e} v_{e t}
$$

$Q_{i e}$ can be further expressed as

$$
Q_{i e}=3 n_{e} m_{e} \kappa\left(T_{e}-T_{i}\right) \sum_{t} \frac{v_{e t}}{m_{e}+m_{t}} .
$$

The heating term accounting for collisions with neutrals consists of two parts: collisional heat transfer and frictional heating:

$$
Q_{i n}=\sum_{t} n_{t} m_{t} \sum_{k} \frac{v_{t k}\left[3 \kappa\left(T_{n}-T_{i}\right)+m_{k}\left(u_{n}-u_{i}\right)^{2}\right]}{m_{t}+m_{k}}
$$

where $n_{t}$ and $m_{t}$ are the number density and mass of ion species $t, u_{n}$ and $u_{i}$ are the neutral and ion velocities, and the subscripts $t$ and $k$ denotes the ion and neutral species respectively. The ion-neutral collision frequencies are given in Appendix B. The corresponding heating rate for the neutrals due to collisions with the ions was added to the heating sources in the neutral energy equation.

\subsection{Numerical Method}

The energy equations are solved using a semi-implicit method, which was described by Huba and Joyce [21]. Schematically, the model uses the backward difference at the current time $t^{n}$ and second-order central difference for the spatial derivative at altitude $z_{j}$ in the conduction calculation at $t^{n}$. Each source term with a linear dependence on the temperature is split into two parts, one with a linear dependence on the variable $T_{j}^{n}$ being solved, and the other without the linear dependence. The linear terms are evaluated at $t^{n}$, while the other terms are evaluated at the previous time step $t^{n-1}$. This semi-implicit method performs well in limiting the stiff source terms, and is numerical stable and convergent.

Both $T_{e}$ and $T_{i}$ are set equal to the neutral temperature at the bottom boundary. $T_{e}$ has a heat flux boundary at the top, such that:

$$
\frac{T_{j}^{n}-T_{j-1}^{n}}{\delta z}=\frac{F_{e}}{\lambda_{e}}
$$




$$
\left(\frac{\partial T^{n}}{\partial z}\right)_{j}=\left(\frac{\partial T^{n}}{\partial z}\right)_{j-1}
$$

Table 1 and 2 present a comparison of the implementation of $T_{e}$ and $T_{i}$ between the old and new model. Four advantages of the new model include: (a) it is capable of simulating $T_{e}$ stably using the same time step as the thermosphere (1-3s); (b) it incorporates the thermoelectric heating in a global I-T model for the first time; (c) it incorporates the horizontal component of the heat conduction along the field lines for $T_{e}$ and $T_{i}$; (d) it allows energy coupling self-consistently between electrons, ions and neutrals.

Table 1. Comparison of $T_{e}$ Calculation between New and Old Methods

\begin{tabular}{|c|c|c|}
\hline Sources(Method) \Model & NEW & OLD \\
\hline Time Derivative & $\sqrt{ }$ & $\times$ \\
Vertical Conduction & $\sqrt{ }$ & $\sqrt{ }$ \\
Horizontal Conduction & $\sqrt{ }$ & $\times$ \\
Photoelectron Heating & $\sqrt{ }$ & $\sqrt{ }$ \\
Auroral Ionization Heating & $\sqrt{ }$ & $\times$ \\
Electron-Ion Collisional Heating & $\sqrt{ }$ & $\sqrt{ }$ \\
Electron-Neutral Elastic Collisional Heating & $\sqrt{ }$ & $\times$ \\
Electron-Neutral Inelastic Collisional Heating & $\sqrt{ }$ & $\sqrt{ }$ \\
Thermoelectric Heating & $\sqrt{ }$ & $\times$ \\
Magnetospheric Heat Flux & $\sqrt{ }$ & $\sqrt{ }$ \\
Two-way Coupling with Ions & $\sqrt{ }$ & $\times$ \\
Two-way Coupling with Neutrals & $\sqrt{ }$ & $\times$ \\
Scheme for Vertical Conduction & Implicit & Implicit \\
Scheme for Other Source Terms & Implicit & Explicit \\
\hline
\end{tabular}

Table 2. Comparison of $T_{i}$ Calculation between New and Old Methods

\begin{tabular}{|c|c|c|}
\hline Sources(Method) \Model & NEW & OLD \\
\hline Time Derivative & $\sqrt{ }$ & $\times$ \\
Vertical Conduction & $\sqrt{ }$ & $\times$ \\
Horizontal Conduction & $\sqrt{ }$ & $\times$ \\
Ion-Electron Collisional Heating & $\sqrt{ }$ & $\times$ \\
Ion-Neutral Elastic Collisional Heating & $\sqrt{ }$ & $\sqrt{ }$ \\
Two-way Coupling with Electrons & $\sqrt{ }$ & $\times$ \\
Two-way Coupling with Neutrals & $\sqrt{ }$ & $\sqrt{ }$ \\
Scheme & Implicit & Steady-State \\
\hline
\end{tabular}




\section{Validation with IRI}

Figure 1 shows comparisons of $N_{e}$ (top), $T_{e}$ (middle) and $T_{i}$ (bottom) using the old model (left), the new model (middle) and the Internation Reference Ionosphere (IRI) model [41] (right) at an altitude of 400 km at 00:00 UT on Dec 23, 2012. The simulation was driven by a constant $F_{10.7}$ index of 110.3 and constant solar wind conditions: $V_{x}=400 \mathrm{~km} / \mathrm{s}$ and $B_{x}=0 \mathrm{nT}, B_{y}=0 \mathrm{nT}$ and $B_{z}=-2 \mathrm{nT}$. The $T_{e}$ produced by the new model was significantly larger than the previous model, especially in the middle and high latitudes on the dayside. Moreover, due to an increase in the ionospheric scale height and a decrease of recombination rates, the magnitude of the ion density in the new model was also increased and had a better agreement with that in IRI. However, the general distributions of the ion densities were similar between the previous and the improved model. The new model reproduced the diurnal variation in the F-region $T_{e}$ at low latitudes, which was characterized by a morning and an evening enhancement. The morning peaks were more pronounced in the winter hemisphere than in the summer hemisphere in both the new model and in IRI, which agrees with Oyama et al. [4]. Compared to IRI, the morning enhancement of the new $T_{e}$ was approximately $500 \mathrm{~K}$ cooler and had a narrower zonal extension at low latitudes. Both the new GITM and IRI show a cooler region of $T_{e}$ in the dayside equatorial region. This feature was caused the inverse correlation between $T_{e}$ and $N_{e}$ in the F-region $[42,43]$. Specifically, the cooling of the electrons exceeds the photoionization heating when $N_{e}$ increases to a certain level [44]. $T_{i}$ in the new model was higher than it was in the old model in both the dayside middle and high latitude regions. The ion Joule heating effect was enhanced in the polar regions in the new model. The new $T_{i}$ has a similar magnitude as IRI on the low-latitude dayside and the south polar region, but was about $500 \mathrm{~K}$ cooler in the north polar region. The difference in $T_{i}$ between the south and north polar regions in GITM was due to the seasonal effect, i.e., the asymmetric solar insolation and geomagnetic forcing [45]. There was more $N_{e}$ in IRI in the northern polar region than in GITM, which would cause a difference in the Joule Heating rate and $T_{i}$.

Figure 2 shows a comparison of electron density (top), electron temperature (middle) and ion temperature (bottom) between the old GITM (left), the new GITM (middle) and IRI (right) at a longitudinal slice of $180^{\circ}$ at 00 UT (i.e., at noon local time). In both the new GITM and IRI, a region filled with denser and cooler electrons existed at low latitudes. However, the topside ionosphere in the new GITM was approximately $500 \mathrm{~K}$ cooler than in IRI in this region. This was due to the inverse relation between $T_{e}$ and $N_{e}$ : a denser ionosphere existed above $400 \mathrm{~km}$ in GITM. Once again, the ion density in the improved model was raised to a more comparable value to IRI due to the increase in $T_{e}$. The distribution of $T_{i}$ in GITM and IRI look very consistent except for the northern polar region, which could be due to the difference in $N_{e}$, thus causing a difference in Joule heating and $T_{i}$ in this region between the two models. 


\section{Validation with Incoherent Scatter Radars}

A time period from April 13th and 14th, 2013 was simulated with the new model when observations from four incoherent scatter radar (ISR) sites were available. The four ISR sites were Arecibo $\left(18.4^{\circ} \mathrm{N}, 66.6^{\circ} \mathrm{W}\right)$, Jicamarca ISR $\left(11.9^{\circ} \mathrm{S}, 76.8^{\circ} \mathrm{W}\right)$, Millstone Hill ISR $\left(40.6^{\circ} \mathrm{N}, 71.5^{\circ} \mathrm{W}\right)$ and Sondrestrom ISR $\left(67.0^{\circ} \mathrm{N}, 50.7^{\circ} \mathrm{W}\right)$. ISRs are capable of measuring the altitudinal profiles of electron density, electron temperature, and ion temperature, to heights of several hundred kilometers by detecting the incoherent backscatter from free electrons in the ionosphere [46]. The simulation was driven by the hemispheric power (HP) obtained by the National Oceanic and Atmospheric Administration (NOAA) POES satellites, as well as the solar wind and interplanetary magnetic field data measured by the Advanced Composition Explorer (ACE), delayed by an appropriate amount of time. The IMF, hemispheric power, and solar wind velocity for April 13 and 14, 2013 are shown in Figure 3. The geomagnetic condition was relatively quiet on April 13th, 2013, with hemispheric power less than $10 \mathrm{GW}$ most of the day, solar wind speed about $400 \mathrm{~km} / \mathrm{s}$ and the magnitude of IMF below 5 nT. On April 14, the hemispheric power and solar wind speed increased to about 40 GW and to about $500 \mathrm{~km} / \mathrm{s}$ early in the day. Both $B_{y}$ and $B_{z}$ turned more positive with larger variability. The $F_{10.7}$ indexes were 125 and 117 on April 13th and 14th, respectively. Figure 4 shows the electron heat flux pattern at the top boundary at 12:00 UT. This pattern was fixed in solar magnetic coordinates but moved in geographic coordinates through the day [31]. The pattern was constructed based on the modeled energy deposition rates from the ring current into the ionospheric thermal electrons during the June 1991 storm [47].

Figure 5 shows a comparison between GITM, IRI and measurement by the Arecibo ISR from $100 \mathrm{~km}$ to $650 \mathrm{~km}$ on April 13th and 14th, 2013. The missing data in the observations were filled by an altitudinal linear interpolation. GITM reproduced the diurnal variation of $T_{e}$ observed by the ISR, but with a weaker morning peak. In both GITM and the ISR data, the morning enhancement occurred at the same local time at all altitudes. However, the local time of the morning enhancement varied with altitudes in IRI. The $T_{e}$ in IRI was about $500 \mathrm{~K}$ higher than GITM and the ISR data. GITM, IRI and the ISR data had a similar diurnal variation of $T_{i}$ below $400 \mathrm{~km}: T_{i}$ began to raise around 6 LT, peaked around $16 \mathrm{UT}$ and decreased to the nightside temperature around $20 \mathrm{LT}$. Above $500 \mathrm{~km}$, GITM underestimated $T_{i}$ by about $300 \mathrm{~K}$ compared to IRI and the ISR data. Also, a distinct morning enhancement in $T_{i}$ was present in GITM, but not IRI or the ISR data.

A comparison between GITM, IRI and the Jicamarca ISR on April 13, 2013 is shown in Figure 6. A morning enhancement in $T_{e}$ existed between $300 \mathrm{~km}$ and $500 \mathrm{~km}$ in GITM, ISI and ISR. However, the morning enhancement in IRI was about $500 \mathrm{~K}$ cooler than GITM and the ISR data. Also, the morning enhancement occurred approximately at the same local time throughout the altitudes in GITM. In IRI, it occurred earlier at higher altitudes, which was opposite of the ISR observation. There were scattered structures of enhanced $T_{e}$ on the nightside in the observations, which were 
not shown in GITM and IRI. This could be equatorial ionospheric perturbations caused by physics that may not be included in the models, or uncertainty in the measurements. The dayside ion temperatures were comparable between the models and the observation. Similar to $T_{e}$, structures of high $T_{i}$ on the nightside were measured by the radar, but were not present in the GITM or IRI.

$T_{e}$ in the F-region highly depends on the downward electron heat flux from the magnetosphere throughout the midand high-latitudes $[12,48,13]$. Two simulations with different heat fluxes specified were conducted to compare the model with mid-latitude observations by the Millstone Hill ISR. Figure 7 shows the diurnal pattern of the heat flux: the small (left y-axis) and large (right y-axis) share the same pattern but with a one order of magnitude difference. The large heat flux was closer to a realistic value of $10^{-5} \mathrm{~W} \mathrm{~m}^{-2}$ [48]. Figure 8 shows the GITM results driven by the small and large electron heat fluxes, with comparison to IRI and the Millstone Hill ISR measurements. The time shown is from 16-24 Local Time, which is from the dashed line to the end of the day in Figure 7. Before about 22 LT, the observed $T_{e}$ was approximately $1000 \mathrm{~K}$ higher than GITM driven by the small heat flux above $400 \mathrm{~km}$. The ion temperature was also underestimated by about $500 \mathrm{~K}$ above $400 \mathrm{~km}$ compared to IRI and the ISR data. $T_{e}$ in IRI had a similar magnitude as observation above $400 \mathrm{~km}$, but was about $1000 \mathrm{~K}$ warmer below.

Driven by the large heat flux, the dayside $T_{e}$ increased significantly above $400 \mathrm{~km}$, which significantly improved the comparison with the observation compared to the small-flux case. Moreover, $T_{i}$ was raised to a similar magnitude as observed by the radar through the heat transfer between electrons and ions. This was especially true at high altitudes, where the collisional heating between electrons and ions is more important. Since a simple empirical heat flux model was used in this simulation, differences between GITM and ISR results can be expected. In order to simulate both $T_{e}$ and $T_{i}$ at mid-latitudes, a well-specified electron heat flux at the top is needed.

Finally, a comparison between GITM, IRI and the Sondrestrom ISR is shown in Figure 9. The heat flux at this location was a constant small value of $10^{-9} \mathrm{~W} \cdot \mathrm{m}^{-2}$ as shown in Figure $4 . T_{e}$ in GITM was smaller than IRI and the ISR data, which could be caused by a few reasons: an underestimation in the driver of particle precipitation, or the fact that the top heat flux specified in the simulation was too low. $T_{i}$ in GITM agreed pretty well with IRI: the $T_{i}$ above $450 \mathrm{~km}$ was about $500 \mathrm{~K}$ higher than the $T_{i}$ below. However, both GITM and IRI underestimated $T_{i}$ around midnight. This could have been caused by not capturing the ion frictional heating term well, which could arise from a non-precise description of the ion or neutral flows in GITM [49]. An underestimation of $T_{e}$ could also lead to a lower value of $T_{i}$, since energy from the electrons flows to the ions through the temperature difference. Further study is needed to determine the high-latitude drivers of the ionospheric temperatures. 


\section{Ionospheric temperature response to an idealized substorm}

GITM with the improved electron and ion energy equations was used to investigate the ionospheric response to an idealized substorm, which was the same as Substorm 4 investigated by Liu and Ridley [30]. The prototypical substorm was constructed based on the superposed epoch variations of IMF $B_{z}$ and HP during substorms using 5years of Challenging Minisatellite Payload (CHAMP) [50] satellite data [1]. The geomagnetic conditions used for the simulation are shown in Figure 10. $B_{z}$ began to decrease at 1 hour before onset, reached a minimum of $-3 \mathrm{nT}$ at -25 min, and recovered to the initial value of zero at 80 minute. The background HP was $20 \mathrm{GW}$. It steadily increased after substorm onset, peaked at $55 \mathrm{GW}$ at $20 \mathrm{~min}$, and recovered to $20 \mathrm{GW}$ at 2 hours epoch time.

In order to explore the effects of just the substorm, two simulations were conducted: one using the idealized substorm input as shown in Figure 10, termed Simulation 1, and the other using constant geomagnetic conditions with $B_{z}=0 \mathrm{nT}$ and $\mathrm{HP}=20 \mathrm{GW}$, termed Simulation 0 . The response to the substorm was expressed as the percentage difference between the two simulations:

$$
T \%=\frac{T_{1}-T_{0}}{T_{0}} \times 100 \%
$$

where $T_{1}$ is a specific ionospheric/thermospheric parameter in Simulation 1 and $T_{o}$ is the parameter in Simulation 0. Figure 11 shows the ionospheric response to the substorm at $140 \mathrm{~km}$ geometrically averaged over the northern polar cap (i.e., above $50^{\circ}$ Latitude). $N_{e}$ had an immediate response to the HP variation due to the small time scale of the E region (i.e., in seconds). The response of $T_{e}$ was highly correlated with the HP, with a cross correlation of 0.99, because the auroral ionization heating was the dominant source for $T_{e}$ and the time scale of the E-region $T_{e}$ modification was small $(\sim \mathrm{s})$. The percentage change of $T_{e}$ peaked around $4 \%$ when the HP reached its maximum value. There was a small enhancement that appeared in $T_{e}$ before the substorm onset. This was due to the ion-electron energy transfer during the growth phase of the substorm. Different than $T_{e}, T_{i}$ started to respond during the growth phase of the substorm when the IMF $B_{z}$ turned southward. The percentage difference of $T_{i}$ peaked at $3 \%$ around the same time when $B_{z}$ became most southward. The IMF-driven electric field was calculated as [51]:

$$
E_{I M F}=V_{s} B_{s} \sin (\theta / 2),
$$

where $V_{s}$ is the solar wind speed in the sunward direction, $B_{s}=\left(B_{y}{ }^{2}+B_{z}{ }^{2}\right)^{\frac{1}{2}}$, and $\theta$ is the angle between the two components of the IMF, $B_{y}$ and $B_{z}$. The response of $T_{i}$ and the square of IMF electric field were highly correlated with a correlation coefficiency of 0.97 . This was because ions in the high-latitude E-region were in a quasi-energy balance with neutrals [28]. With this assumption, $T_{i}$ can be approximated as [2, 17]: 


$$
T_{i}=T_{n}+\frac{m_{n}}{3 \kappa}\left(\boldsymbol{u}_{n}-\boldsymbol{u}_{i}\right)^{2}
$$

The estimated $T_{i}$ was dependent on the neutral temperature and the difference in bulk velocity between the ions and neutrals. The responding profiles of the estimated $T_{i}$ and the simulated $T_{i}$ had a high correlation of 0.99 . The estimated $T_{i}$ was always cooler than the simulated $T_{i}$ because the energy balance assumption was not completely accurate for ions in the E region where electrons were sufficiently dense to affect the ion temperature through heat transfer. The temporal profile of $T_{i}$ variation was quite similar to the variation of $\delta V^{2}$, which is $\left(\boldsymbol{u}_{\boldsymbol{n}}-\boldsymbol{u}_{\boldsymbol{i}}\right)^{2}$. This indicates that the velocity difference between the ions and neutrals, which represents the ion-neutral collisional heating, was the dominant heat source for ions during the substorm. As shown in the bottom panel in Figure 11, the variation of the velocity difference was controlled by the IMF-induced electric field. This was expected because the IMF electric field driver changed the ion drift significantly, while the neutral winds did not change much during the substorm [52]. During the recovery phase of the substorm (i.e., about $60 \mathrm{~min}$ ), the neutrals started to ramp up [30] affecting the velocity difference and altering $T_{i}$.

Figure 12 shows the spatial variation of the ionospheric response at 6 times around the substorm onset. The leftmost column shows the background ionospheric state 60 min before the substorm onset, while the after plots show perturbations of the state variables with respect to Simulation 0 . The perturbation in $N_{e}$ was relatively small until after the substorm onset. $N_{e}$ was enhanced by $\sim 100 \%$ within the auroral oval when the HP reached maximum value. The red bands on the inner and outer boundaries were due to an expansion of the auroral oval and a low $N_{e}$ background in these regions. Similar to $N_{e}, T_{e}$ did not show noticeable perturbation until the HP increased when the auroral heating increased $T_{e}$ by about $25 \%$ in the auroral oval. The perturbation in $T_{i}$ started during the growth phase of the substorm as a response to the southward turning of IMF. The maximum enhancement occurred in the low-latitude convection zone, where large shears existed between the day-to-night neutral wind and the sunward ion flows. The perturbance died down before the onset time when $B_{z}$ recovered.

Figure 13 shows the ionospheric response at $300 \mathrm{~km}$ in the same format as Figure 11. The response of $N_{e}$ at $300 \mathrm{~km}$ was relatively sluggish compared to the response at $140 \mathrm{~km}$. The cross correlation between HP and the $N_{e}$ perturbation peaked at 0.78 with a time delay of 30 minutes. This is because $\sim \mathrm{keV}$ precipitating electrons mainly deposited their energy in the E-region [53]. Three factors could account for the enhancement of $N_{e}$ in the F-region: (a) an increase of $T_{e}$ led to a decrease in the dissociative recombination rate, which is discussed in detail below; (b) plasma was transported from the E-region due to diffusion along the magnetic field; (c) the lower energy precipitating particles in the distribution caused some ionization in the F-region. Further investigation is needed to determine how these processes combine to influence the response of the F-region. However, the relatively large chemical time scale 
of the ions in the F-region led to a long production and life time of the electron density perturbation in the F-region.

As shown in the second panel in Figure 13, the response of $T_{e}$ was in phase with the variation of the HP, which suggested that a major source of $T_{e}$ in the $\mathrm{F}$ region was heat conducted from the E region since there was little auroral heating in the F-region. The fact that the F-region temperature was highly correlated with the E-region density instead of the F-region density was also observed by Maehlum et al. [26] and Wahlund et al. [54] during an intense aurora observed by EISCAT. A slightly negative perturbation of $T_{e}$ occurred between 1 hour and 4 hour epoch time. What probably happened was that the heat conduction was into the F-region during the first part of the substorm and then out of the F-region during the second part of the substorm.

The estimate $T_{i}$ was quite similar to the simulated $T_{i}$ during the substorm, which indicates that the electron-ion heat transfer was negligible in the high-latitude F-region [2], and that the ion-neutral coupling was the dominant process. The major heat source to $T_{i}$ was ion Joule heating and the major cooling term was heat transfer to neutrals. Before the substorm onset, the ion Joule heating driven by the IMF electric field primarily contributed to the enhancement of $T_{i}$. After 0 hour epoch time, the neutral temperature gradually increased, which reduced the speed of the cooling process of $T_{i}$. A slight difference between the simulated and estimate $T_{i}$ appeared between 0 and 1.5 hours because the electron-ion heat transfer was enhanced due to an increase in $N_{e}$ and $T_{e}$ after the substorm onset.

Figure 14 shows the spatial distribution of the F-region ionospheric perturbation in the same format as Figure 12. A prominent increase in $N_{e}$ appeared after HP reached a maximum. At 60 min, a perturbation of about $100 \%$ existed around the auroral oval, as well as a bright band of about $300 \%$ on the outer boundary. The width of the perturbed region was about 4 times the width of the background auroral oval, similar to what was observed in the E-region. The F-region perturbation lasted for more than 2 hours due to relatively slow charge exchange and recombination rates. The response of $T_{e}$ in the F-region was similar to the E-region. The percentage change in $T_{e}$ reached about $30 \%$ in the main oval and around the high-latitude boundary of the auroral oval, and about $60 \%$ near the low-latitude boundary of the auroral oval when HP peaked at $20 \mathrm{~min}$. The response of $T_{i}$ maximized at -25 min when the IMF was most southward. The ion temperature then gradually died down after the peak.

\section{Heat flux effect}

In order to investigate the influence of the electron heat flux on the ionosphere, two simulations of the idealized substorm (as shown in Figure 10) were conducted, one with a small heat flux and one with a large heat flux during the substorm. The heat flux distributions were the same, but the large heat flux was one order of magnitude larger than the smaller heat flux, as shown in Figure 4. Figure 15 shows the ionospheric response to the two different heat fluxes. The increased heat flux was most effective on increasing $T_{e}$ on the nightside in the mid-latitudes where $N_{e}$ 
was approximately 100 times smaller than on the dayside. $T_{e}$ was enhanced by $\sim 100 \%$ at maximum in the dusk and midnight regions. No obvious enhancement in $T_{e}$ existed on the dayside. The increase in the heat flux also raised $N_{e}$ and TEC, especially on the nightside. The enhancement in the electron density reached $\sim 80 \%$ at maximum, but occurred in the region in which the density was very low.

Figure 16 shows vertical profiles of $T_{e}$ (left) and $N_{e}$ (right) at $150^{\circ}$ Lon and $55^{\circ}$ Lat at 1200 UT (i.e., $2200 \mathrm{LT}$ ), which is in the middle of the electron heat flux region on the nightside. The dotted and the solid lines represent the simulation results with the small and large heat fluxes, respectively. The increase in the heat flux raised $T_{e}$ above approximately $140 \mathrm{~km}$. The enhancement increased with altitude, and reached over $1000 \mathrm{~K}$ at $600 \mathrm{~km}$. $N_{e}$ was also increased above $140 \mathrm{~km}$. The enhancement in $N_{e}$ due to the increase in the heat flux was about $3 \times 10^{9} \mathrm{~m}^{-3}(\sim 60 \%)$ above $230 \mathrm{~km}$. This result agreed well with David et al. [13].

The fact that the increase in the heat flux increased $N_{e}$ was due to the combination of three factors in the ionosphere [2]:

1. An increase in $T_{e}$ can increase the ionospheric scale height, which reduces the exponential decrease of $N_{e}$ with altitude above the F-region peak.

2. An increase in $T_{e}$ can raise $T_{i}$ through the electron-ion collisional heat transfer. The increase of $T_{i}$ accelerates the charge exchange ratio between $\mathrm{O}^{+}$and molecular neutrals $\mathrm{N}_{2}, \mathrm{O}_{2}$ and $\mathrm{NO}$. As the dissociative recombination rates of the resulting molecular ions are approximately five orders of magnitude faster than the radiative recombination rates of $O^{+}$, the increase in $T_{e}$ could resulted in a decrease of $N_{e}$ by raising $T_{i}$.

3. The radiative and dissociative recombination rates of atomic and molecular ion, respectively, are inversely proportional to $T_{e}$. Therefore, an increase of $T_{e}$ could lead to an increase of $N_{e}$ by reducing the recombination rates.

Here, a simple calculation was done to determine the net effect of changing either the electron or ion temperature on the F-region density due to the charge exchange and dissociative recombination reactions. All the other ionospheric processes, such as transport and photoionization, were ignored.

Three charge exchange reactions were considered:

$$
\begin{gathered}
O^{+}+N_{2} \stackrel{\kappa_{1}}{\rightarrow} N O^{+}+N, \\
O^{+}+O_{2} \stackrel{\kappa_{2}}{\rightarrow} O_{2}^{+}+O,
\end{gathered}
$$




$$
\mathrm{O}^{+}+\mathrm{NO} \stackrel{\kappa_{3}}{\rightarrow} \mathrm{NO}^{+}+\mathrm{O}
$$

where the reaction rates $\kappa_{1}, \kappa_{2}$, and $\kappa_{3}$ are $T_{i}$ dependent, and given in Appendix C. Two dissociative recombination reactions and the $O^{+}$radiative recombination reaction were considered:

$$
\begin{gathered}
N O^{+}+e^{-} \stackrel{\kappa_{4}}{\rightarrow} O+N, \\
O_{2}^{+}+e^{-} \stackrel{\kappa_{5}}{\rightarrow} O+O, \\
O^{+}+e^{-} \stackrel{\kappa_{6}}{\rightarrow} O
\end{gathered}
$$

where the reaction rates $\kappa_{4}, \kappa_{5}, \kappa_{6}$ are $T_{e}$ dependent, and given in Appendix C. The initial ion densities (in $\mathrm{m}^{-3}$ ) were set as: $n\left(O^{+}\right)=1.0 \times 10^{12}, n\left(N O^{+}\right)=0.0$ and $n\left(O_{2}^{+}\right)=0.0$. The initial neural densities (in $\mathrm{m}^{-3}$ ) were set as $n(N O)=1.0 \times 10^{10}, n\left(O_{2}\right)=1.0 \times 10^{11}$ and $n\left(N_{2}\right)=1.0 \times 10^{12}$. The background $O+$ number density and neutral densities were typical F-region values from the simulation. Two steps were taken: (a) the charge exchange reactions were processed for one second, converting $\mathrm{O}^{+}$to $\mathrm{NO}^{+}$and $\mathrm{O}_{2}^{+}$; and (b) the recombination reactions were then processed for one second. The ion loss rate was obtained by subtracting the initial total ion density from the final total ion density. The calculation was conducted in the range of: $T_{e} \sim[1000 \mathrm{~K}, 6000 \mathrm{~K}]$ and $T_{i} \sim[1000 \mathrm{~K}$, $3000 \mathrm{~K}]$. The loss rate was normalized at the state of $T_{e}=1000 \mathrm{~K}$ and $T_{i}=1000 \mathrm{~K}$, as given in Figure 17 . The highest ion loss rate existed in the up left corner (i.e., ion temperature much larger than electron temperature), where both the charge exchange rates were most enhanced but the dissociative recombination rates were most reduced. This could happened in regions with strong Joule heating, raising $T_{i}$ even higher than $T_{e}[44,12]$. A strong decrease in ion density would be expected in such a region. Likewise, the lowest ion loss rate occurred in the lower right corner (i.e., electron temperature much larger than ion temperature), where the charge exchange rates were mostly suppressed, but the dissociative recombination rates were enhanced. This circumstance can be expected in regions with strong downward electron heat flux [13] or upward field-aligned currents, where $T_{e}$ could be significantly increased [12, 14]. The GITM simulation results shown in Figure 16 showed a positive relationship between $T_{e}$ and $N_{e}$, which indicates that the effects of $T_{e}$ on $N_{e}$ caused by the change of the dissociative recombination rates (and the change of the ionospheric scale height) were dominant over the change of the charge exchange rates. 


\section{Summary and Conclusion}

The improvement of the electron and ion temperature calculation in the Global Ionosphere Thermosphere Model has been described in this paper. Time-dependent energy equations for $T_{e}$ and $T_{i}$ with heating and cooling rates, as well as field-aligned thermal conduction were implemented. The sources of energy in the electron equation include heating due to photoionization and ionization due to particle precipitation, elastic collisions with ions, elastic and inelastic collisions with neutrals, as well as energy flux from the ring current/plasmasphere interaction. The sources of the ion temperature equation include elastic collisions with electrons and neutrals as well as frictional heating with neutrals.

The new GITM produced higher $T_{e}$ and $T_{i}$ at middle and high latitudes than the old model, which led to a denser ionosphere that was more comparable to the ionosphere in IRI. The new GITM reproduced the global structure of $T_{e}$ observed in IRI including morning and evening enhancements at low and mid-latitudes. An expected anti-correlation between $T_{e}$ and $N_{e}$ was also observed in the new model. The temporal variations of the vertical distribution of $T_{e}$ and $T_{i}$ were similar to the Arecibo ISR observations. Compared to the observations by the Jicamarca ISR, GITM generally captured the magnitudes and the diurnal variations of $T_{e}$ and $T_{i}$, but missed the small-scale structures on the nightside. It was also found that the mid-latitude $T_{e}$ and $T_{i}$ were strongly related to the electron heat flux. GITM needed a larger, more realistic heat flux input at the top boundary in order to reproduce similar magnitudes of $T_{e}$ and $T_{i}$ compared to the Millstone ISR observations. GITM also reproduced the general variations observed by the Sondrestrom ISR, but underestimated $T_{e}$ and $T_{i}$, which was probably due to the lack of the heat flux in the auroral oval in the model and a lack of proper electric field description to capture the realistic frictional heating.

The ionospheric response to an idealized substorm was investigated using the new version of GITM. The variation in $N_{e}$ and $T_{e}$ in the E-region were highly correlated with the variation in HP due to rapid auroral ionization processes and the short chemical time scale $(\sim \mathrm{s})$ in this region. $T_{i}$ began to respond during the growth phase of the substorm when the IMF $B_{z}$ turned southward. The variation of $T_{i}$ was mainly controlled by the IMF-driven electric field because the ion Joule heating rate was dependent on the ion velocity and the change of ion drift was mainly driven by the IMF, assuming a relatively steady neutral wind pattern. The response of the F-region $T_{e}$ was not correlated with the Fregion $N_{e}$, but was correlated with the E-region $T_{e}$ and $N_{e}$, which is consistent with observations by Maehlum et al. [26] and Wahlund et al. [54]. This was because there was little auroral deposition in the F-region. The electron thermal conduction efficiently and rapidly transported heat from the E-region to the F-region, while the time scale of the electron density variations in the F-region was hours. The variations of the F-region $T_{i}$ were also dominated by the IMF-driven electric field through the ion flow velocity and the frictional energy coupling with the neutrals.

The increase of the topside heat flux by one order of magnitude in the mid-latitudes raised $T_{e}, N_{e}$ and TEC by 

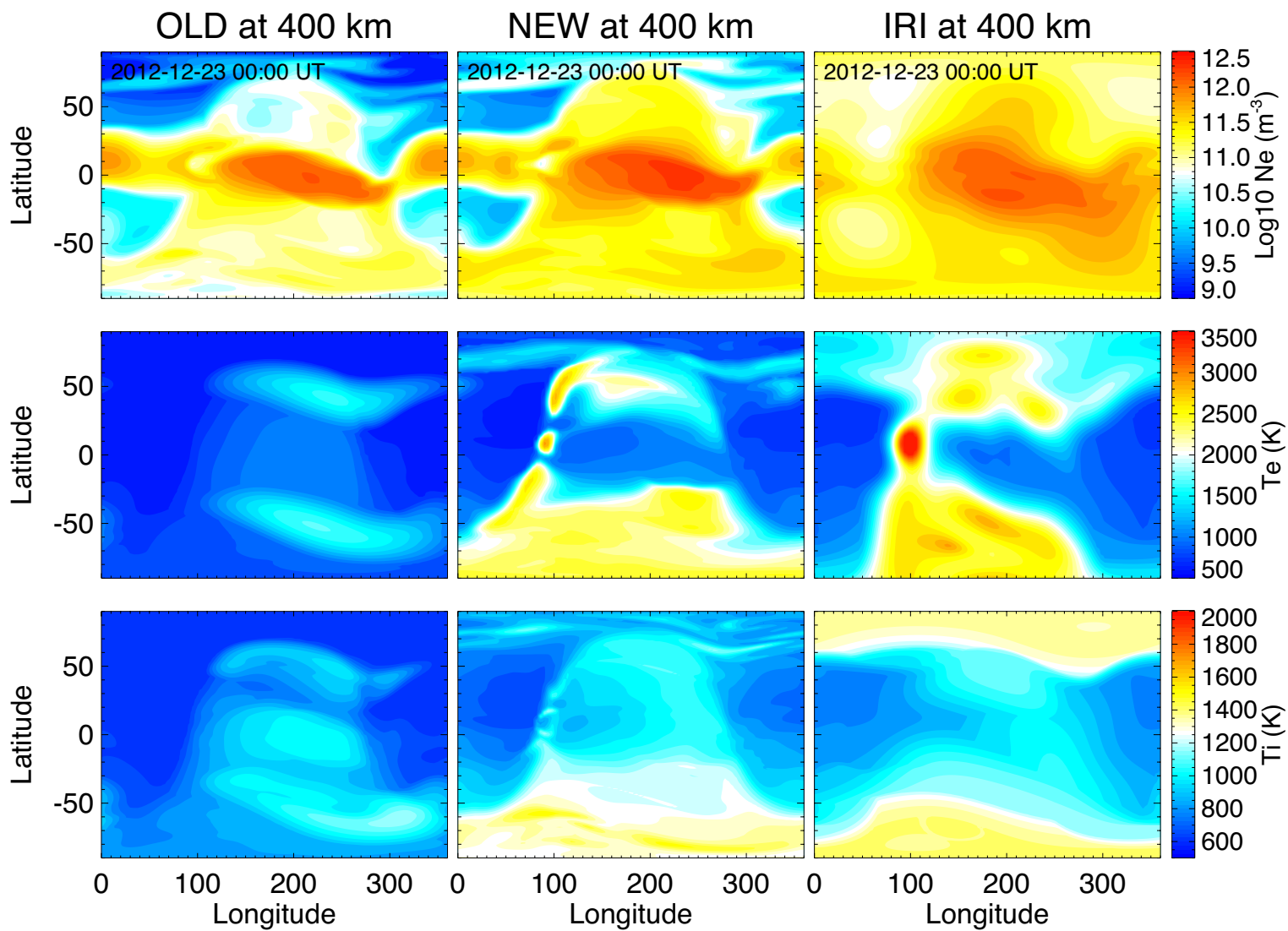

Figure 1. Comparisons of electron density (top), electron temperature (mid) and ion temperature (bottom) among the old GITM, the new GITM old and IRI at an altitudinal cut at $400 \mathrm{~km}$ at 00:00 UT on Nov 25, 2012.

approximately $120 \%, 80 \%$ and $80 \%$ (maximum), respectively in the dusk-to-midnight sector. The influence of the increased heat flux on the nightside penetrated down to about $140 \mathrm{~km}$. The dayside ionosphere did not show significant changes in any of the variables in the increased heat flux region, due to the large electron density. The charge exchange and dissociative recombination reactions, which are $T_{i}$ and $T_{e}$ dependent respectively, are the two important chemical processes controlling the loss rate of the F-region ionosphere [2]. High $T_{e}$ and relatively low $T_{i}$ tend to reduce the F-region ion loss rate, which can cause the F-region density in the heat flux region to reduce at a slower rate than it would with minimal heat flux.

\section{Appendix A. Source Terms in the Calculation of the Electron Temperature}

The electron thermal conductivity $\lambda_{e}$ is given by Schunk and Nagy [2]:

$$
\lambda_{e}=\frac{7.7 \times 10^{7} T_{e}^{5 / 2}}{1+3.22 \times 10^{8} \frac{T_{e}^{2}}{n_{e}} \Sigma_{n} n_{n}<Q_{e n}^{(1)}>},
$$




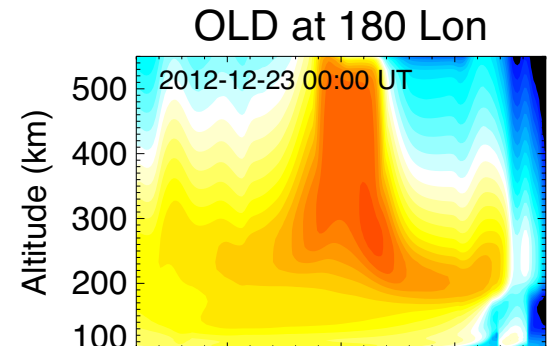

NEW at 180 Lon
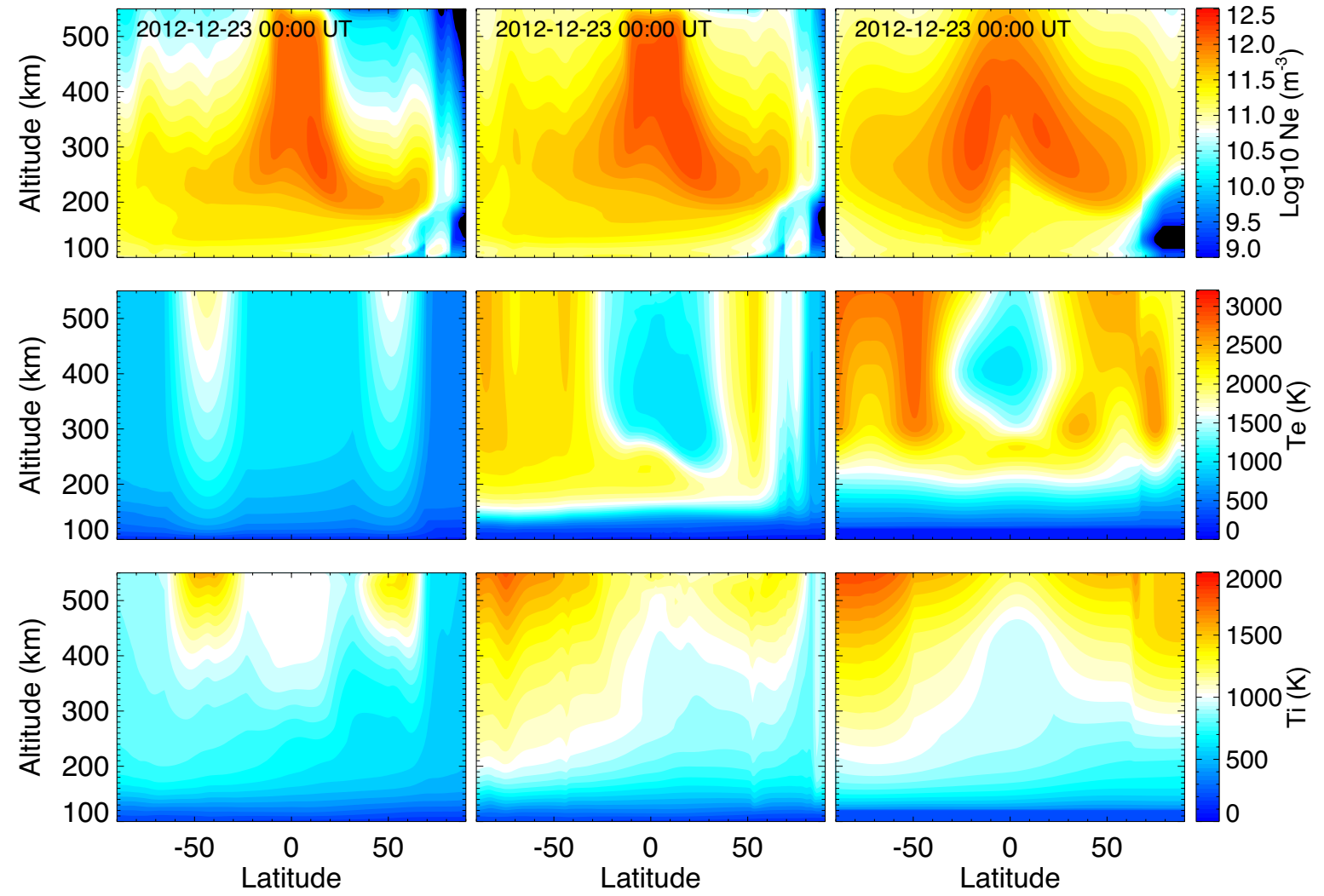

Figure 2. Comparisons of electron density (top), electron temperature (mid) and ion temperature (bottom) among the old GITM, the new GITM and IRI at a longitudinal cut at $180^{\circ}$ at 00:00 UT on Nov 25, 2012.

where $T_{e}, n_{e}$ and $n_{n}$ are the electron temperature, electron density and neutral densities, respectively. The densities are in $\mathrm{m}^{-3}$, and $\lambda_{e}$ is in $\mathrm{eV} \mathrm{m}^{-1} \mathrm{~s}^{-1} \mathrm{~K}^{-1} .<Q_{e n}^{(1)}>$ is a Maxwellian average of the momentum transfer cross section, which was simplified as $1.0 \times 10^{-20} \mathrm{~m}^{2}$ for all the neutral species in the model. For the theoretical momentum transfer cross sections for some neutral species (not used here), please refer to [55].

The electron-ion collision frequency is given by Schunk and Nagy [2]:

$$
v_{e t}=5.45 \times 10^{-5} \frac{n_{t} Z_{t}^{2}}{T_{e}^{3 / 2}},
$$

444

where $n_{t}$ is the number density of ion species $t$ in $\mathrm{m}^{-3}$ and $Z_{t}$ is the particle charge number.

The electron-neutral collision frequencies are given by [56, 57]:

$$
v_{e n}(O)=8.9 \times 10^{-17} n(O)\left(1+5.7 \times 10^{-4} T_{e}\right) T_{e}^{1 / 2},
$$




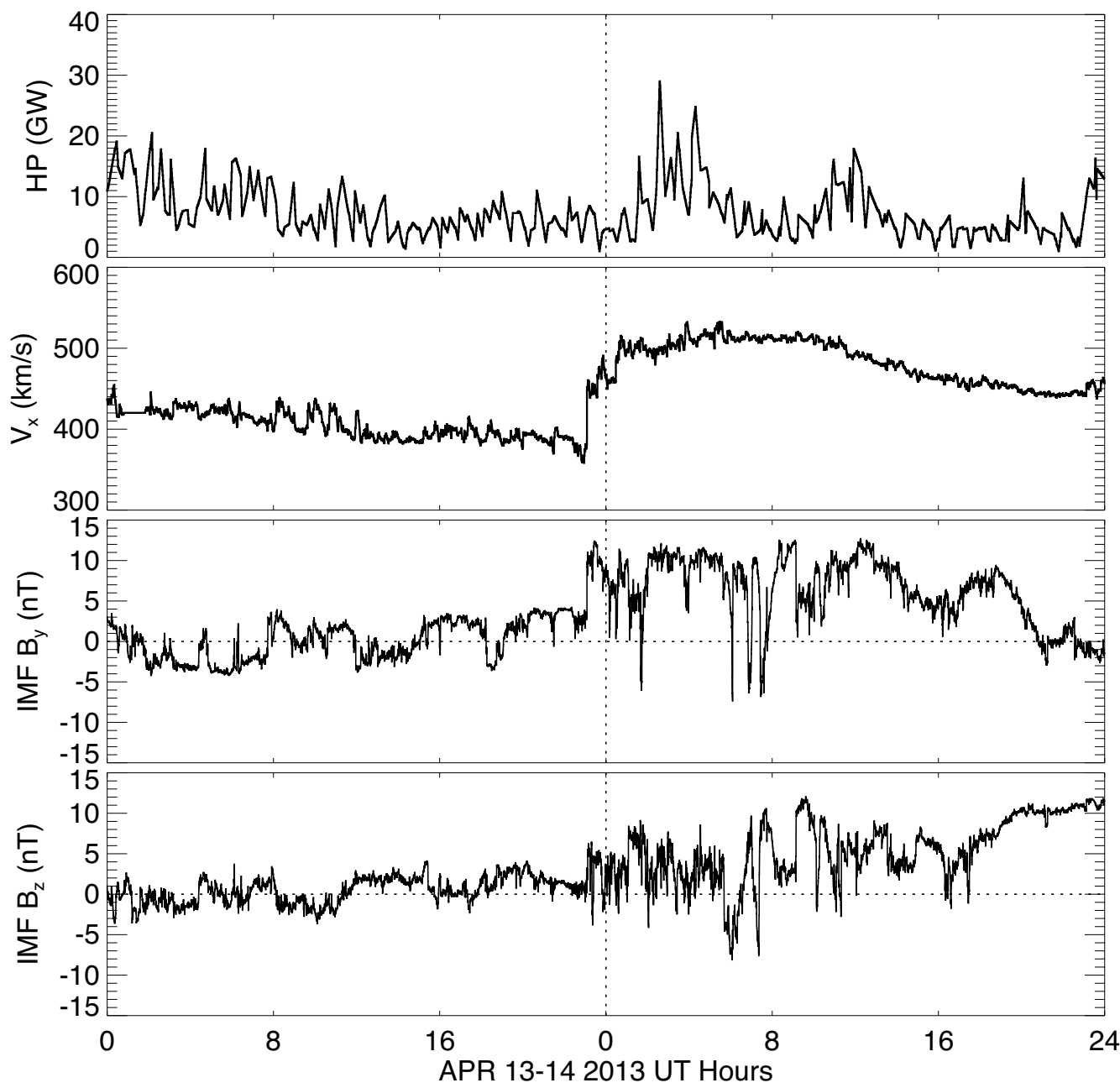

Figure 3. Temporal variations of hemispheric power, solar wind velocity Vx, IMF By and IMF Bz in GSE coordinates from top to bottom on April $13-14,2013$.

$$
\begin{gathered}
v_{e n}\left(N_{2}\right)=2.33 \times 10^{-17} n\left(N_{2}\right)\left(1-1.21 \times 10^{-4} T_{e}\right) T_{e}, \\
v_{e n}\left(O_{2}\right)=1.82 \times 10^{-16} n\left(O_{2}\right)\left(1+3.6 \times 10^{-2} T_{e}^{1 / 2}\right) T_{e}^{1 / 2},
\end{gathered}
$$
respectively. The neutral densities are in $\mathrm{m}^{-3}$.

The electron cooling rates in $\mathrm{eV} \mathrm{m}^{-3} \mathrm{~s}^{-1}$ due to inelastic collisions with neutrals are given by the following expressions (densities in $\mathrm{m}^{-3}$ ):

$\mathbf{N}_{2}$ rotation [58]: 


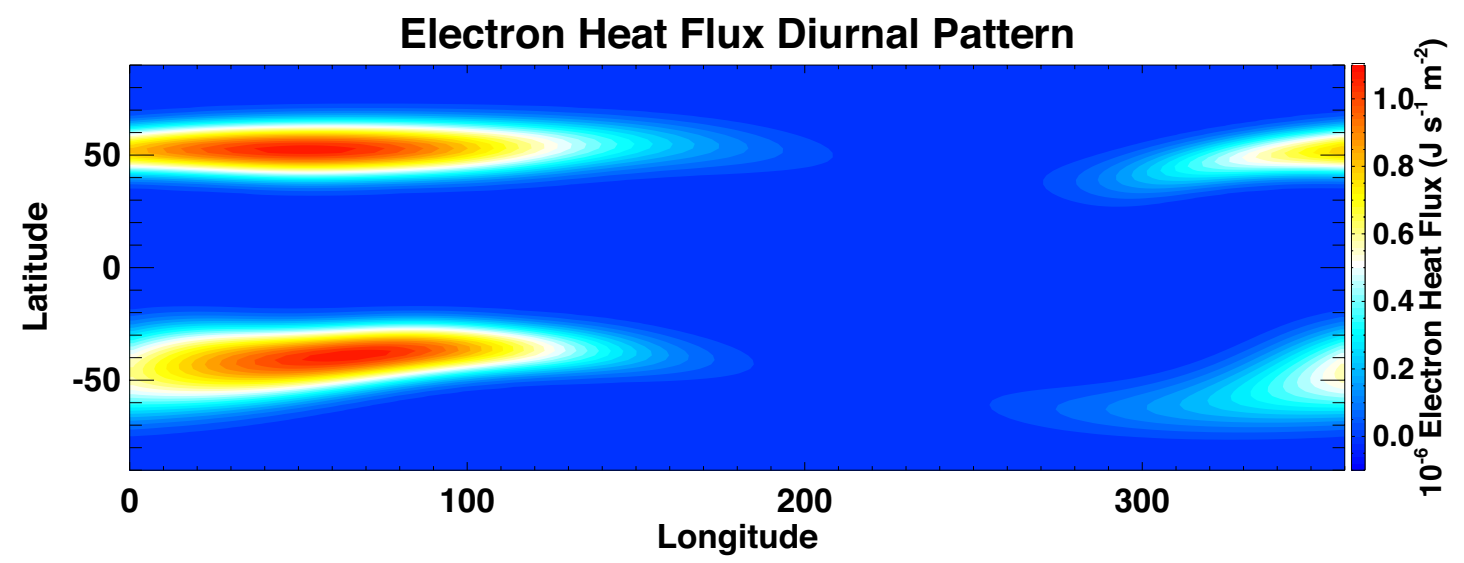

Figure 4. Global distribution of the electron heating flux at the top boundary at 12:00 UT.

$$
L_{e}\left(N_{2}\right)_{r o t}=3.5 \times 10^{-26} n_{e} n\left(N_{2}\right)\left(T_{e}-T_{n}\right) T_{e}{ }^{1 / 2} .
$$

$\mathbf{O}_{2}$ rotation [59]:

$$
L_{e}\left(O_{2}\right)_{r o t}=5.2 \times 10^{-27} n_{e} n\left(O_{2}\right)\left(T_{e}-T_{n}\right) T_{e}^{1 / 2} .
$$

$\mathbf{N}_{2}$ vibration [58]:

$$
\begin{gathered}
L_{e}\left(N_{2}\right)_{v i b}=1 \times 10^{-12} n_{e} n\left(N_{2}\right)\left(1-e^{\left(-E_{1} / T_{v i b}\right)}\right) \\
\sum_{v=1}^{10} Q_{0 v}\left(1-e^{\left(v E_{1}\left(T_{e}^{-1}-T_{v i b}^{-1}\right)\right)}\right) \\
+1 \times 10^{-12} n_{e} n\left(N_{2}\right)\left(1-e^{\left(-E_{1} / T_{v i b}\right)}\right) e^{\left(-E_{1} / T_{v i b}\right)} \\
\sum_{v=2}^{9} Q_{1 v}\left(1-e^{\left((v-1) E_{1}\left(T_{e} e^{-1}-T_{v i b}{ }^{-1}\right)\right)}\right),
\end{gathered}
$$

where $E_{1}=3353 \mathrm{~K}, T_{v i b}=T_{n}$ and

$$
\begin{aligned}
& \log _{10} Q_{0 v}=A_{0 v}+B_{0 v} T_{e}+C_{0 v} T_{e}^{2}+D_{0 v} T_{e}^{3}+F_{0 v} T_{e}^{4}-16 \\
& \log _{10} Q_{1 v}=A_{1 v}+B_{1 v} T_{e}+C_{1 v} T_{e}^{2}+D_{1 v} T_{e}^{3}+F_{1 v} T_{e}^{4}-16 .
\end{aligned}
$$

The A, B, C, D and F coefficients are given in Tables A.3, A.4, and A.5. 


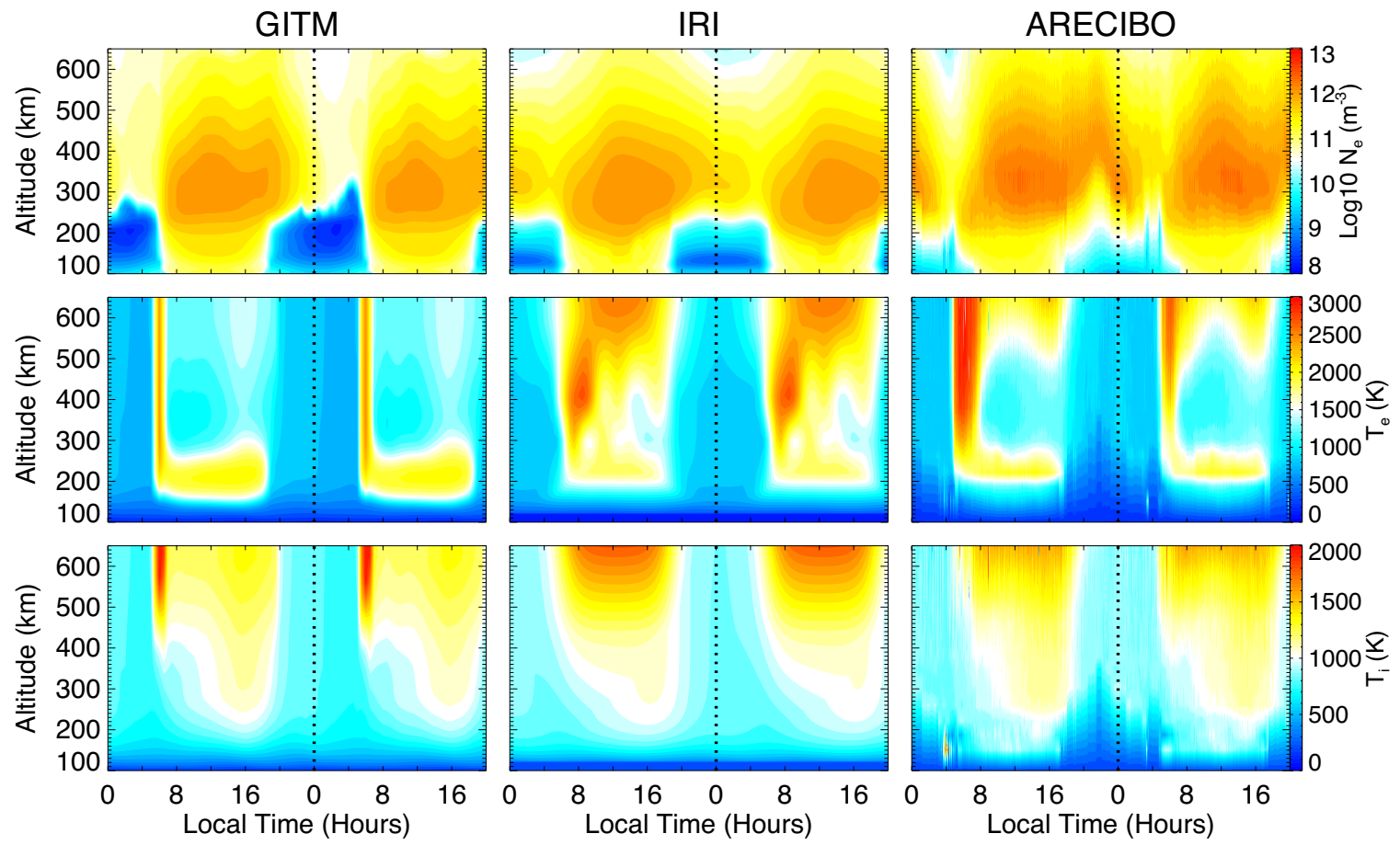

Figure 5. Comparisons of temporal variations in the electron density (top), electron temperature (mid) and ion temperature (bottom) between GITM (left column), IRI (right column) and observation by the Arecibo ISR (right column) from $100 \mathrm{~km}$ to $650 \mathrm{~km}$ on April 13 th and 14th, 2013.

Table A.3. Coefficients for calculations of $Q_{0 v}$ for $300 \leq T_{e} \leq 1500 \mathrm{~K}$

\begin{tabular}{|c|c|c|c|c|c|c|}
\hline \hline$v$ & $A_{0 v}$ & $B_{0 v}, K^{-1}$ & $C_{0 v}, K^{-2}$ & $D_{0 v}, K^{-3}$ & $F_{0 v}, K^{-4}$ & $\delta_{0 v}$ \\
\hline 1 & -6.462 & $3.151 \cdot 10^{-2}$ & $-4.075 \cdot 10^{-5}$ & $2.439 \cdot 10^{-8}$ & $-5.479 \cdot 10^{-12}$ & 0.14 \\
\hline \hline
\end{tabular}

Table A.4. Coefficients for calculations of $Q_{0 v}$ for $1500 \leq T_{e} \leq 6000 \mathrm{~K}$

\begin{tabular}{|l|c|c|c|c|c|c|}
\hline \hline$v$ & $A_{0 v}$ & $B_{0 v}, K^{-1}$ & $C_{0 v}, K^{-2}$ & $D_{0 v}, K^{-3}$ & $F_{0 v}, K^{-4}$ & $\delta_{0 v}$ \\
\hline 1 & 2.025 & $8.782 \cdot 10^{-4}$ & $2.954 \cdot 10^{-7}$ & $-9.562 \cdot 10^{-11}$ & $7.252 \cdot 10^{-15}$ & 0.06 \\
\hline 2 & -7.066 & $1.001 \cdot 10^{-2}$ & $-3.066 \cdot 10^{-6}$ & $4.436 \cdot 10^{-10}$ & $-2.449 \cdot 10^{-14}$ & 0.08 \\
\hline 3 & -8.211 & $1.092 \cdot 10^{-2}$ & $-3.369 \cdot 10^{-6}$ & $4.891 \cdot 10^{-10}$ & $-2.706 \cdot 10^{-14}$ & 0.10 \\
\hline 4 & -9.713 & $1.204 \cdot 10^{-2}$ & $-3.732 \cdot 10^{-6}$ & $5.431 \cdot 10^{-10}$ & $-3.008 \cdot 10^{-14}$ & 0.10 \\
\hline 5 & -10.353 & $1.243 \cdot 10^{-2}$ & $-3.850 \cdot 10^{-6}$ & $5.600 \cdot 10^{-10}$ & $-3.100 \cdot 10^{-14}$ & 0.13 \\
\hline 6 & -10.819 & $1.244 \cdot 10^{-2}$ & $-3.771 \cdot 10^{-6}$ & $5.385 \cdot 10^{-10}$ & $-2.936 \cdot 10^{-14}$ & 0.15 \\
\hline 7 & -10.183 & $1.185 \cdot 10^{-2}$ & $-3.570 \cdot 10^{-6}$ & $5.086 \cdot 10^{-10}$ & $-2.769 \cdot 10^{-14}$ & 0.15 \\
\hline 8 & -12.698 & $1.309 \cdot 10^{-2}$ & $-3.952 \cdot 10^{-6}$ & $5.636 \cdot 10^{-10}$ & $-3.071 \cdot 10^{-14}$ & 0.15 \\
\hline 9 & -14.710 & $1.409 \cdot 10^{-2}$ & $-4.249 \cdot 10^{-6}$ & $6.058 \cdot 10^{-10}$ & $-3.300 \cdot 10^{-14}$ & 0.15 \\
\hline 10 & -17.538 & $1.600 \cdot 10^{-2}$ & $-4.916 \cdot 10^{-6}$ & $7.128 \cdot 10^{-10}$ & $-3.941 \cdot 10^{-14}$ & 0.15 \\
\hline \hline
\end{tabular}

\section{$\mathbf{O}_{2}$ vibration [60]:}

$$
L_{e}\left(O_{2}\right)_{v i b}=1 \times 10^{-12} n_{e} n\left(O_{2}\right) Q\left(T_{e}\right)\left(1-e^{\left(2239\left(T_{e}^{-1}-T_{n}^{-1}\right)\right)}\right),
$$




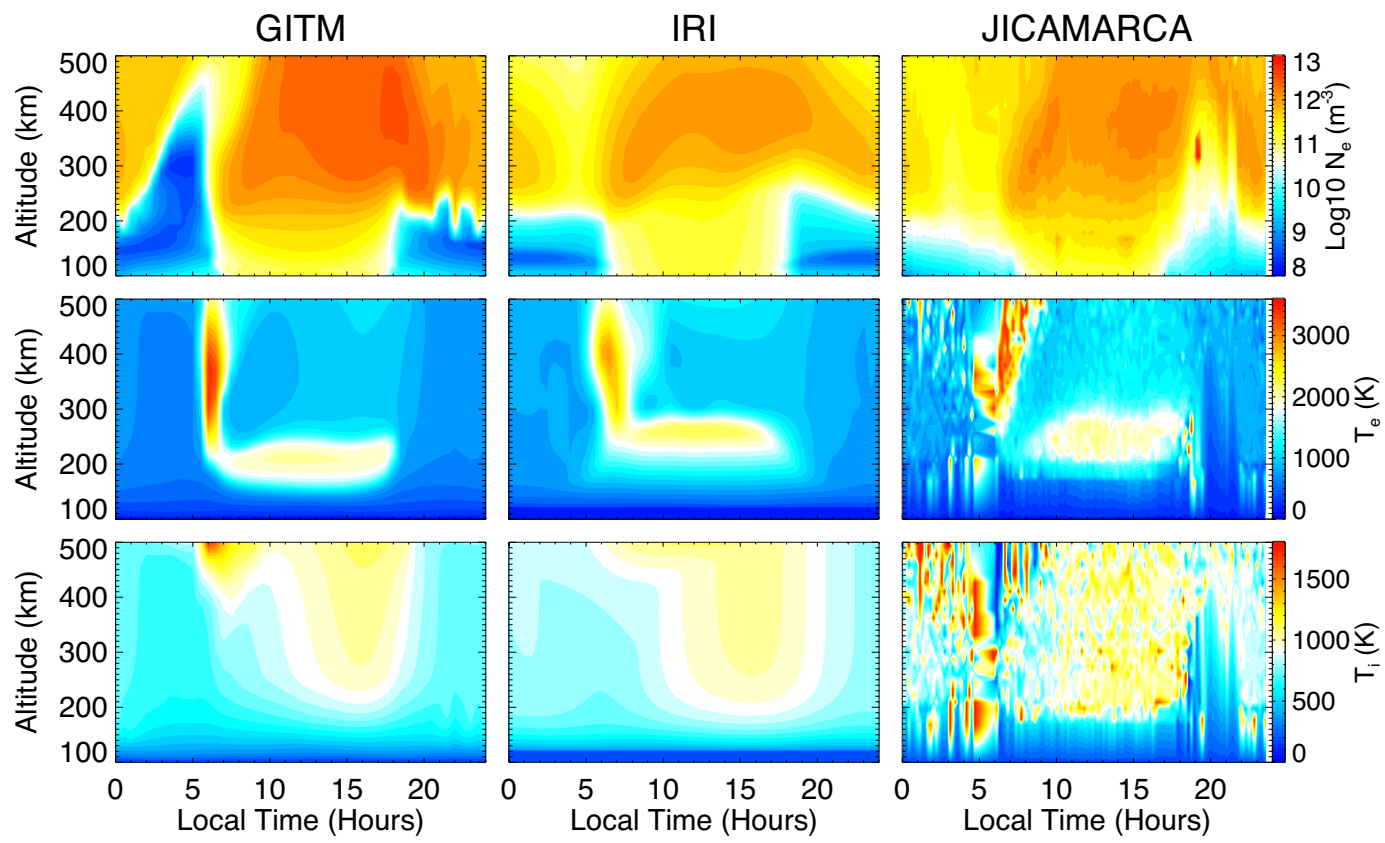

Figure 6. Comparisons of temporal variations in the electron density (top), electron temperature (mid) and ion temperature (bottom) between GITM (left column), IRI (center column) and observation by the Jicamarca ISR (right column) from $100 \mathrm{~km}$ to $650 \mathrm{~km}$ on April 13 th, 2013.

Table A.5. Coefficients for calculations of $Q_{1 v}$ for $1500 \leq T_{e} \leq 6000 \mathrm{~K}$

\begin{tabular}{|c|c|c|c|c|c|c|}
\hline \hline$v$ & $A_{1 v}$ & $B_{1 v}, K^{-1}$ & $C_{1 v}, K^{-2}$ & $D_{1 v}, K^{-3}$ & $F_{1 v}, K^{-4}$ & $\delta_{1 v}$ \\
\hline 2 & -3.413 & $7.326 \cdot 10^{-3}$ & $-2.200 \cdot 10^{-6}$ & $3.128 \cdot 10^{-10}$ & $-1.702 \cdot 10^{-14}$ & 0.11 \\
\hline 3 & -4.160 & $7.803 \cdot 10^{-3}$ & $-2.352 \cdot 10^{-6}$ & $3.352 \cdot 10^{-10}$ & $-1.828 \cdot 10^{-14}$ & 0.11 \\
\hline 4 & -5.193 & $8.360 \cdot 10^{-3}$ & $-2.526 \cdot 10^{-6}$ & $3.606 \cdot 10^{-10}$ & $-1.968 \cdot 10^{-14}$ & 0.12 \\
\hline 5 & -5.939 & $8.807 \cdot 10^{-3}$ & $-2.669 \cdot 10^{-6}$ & $3.806 \cdot 10^{-10}$ & $-2.073 \cdot 10^{-14}$ & 0.08 \\
\hline 6 & -8.261 & $1.010 \cdot 10^{-2}$ & $-3.039 \cdot 10^{-6}$ & $4.318 \cdot 10^{-10}$ & $-2.347 \cdot 10^{-14}$ & 0.10 \\
\hline 7 & -8.185 & $1.010 \cdot 10^{-2}$ & $-3.039 \cdot 10^{-6}$ & $4.318 \cdot 10^{-10}$ & $-2.347 \cdot 10^{-14}$ & 0.12 \\
\hline 8 & -10.823 & $1.199 \cdot 10^{-2}$ & $-3.620 \cdot 10^{-6}$ & $5.159 \cdot 10^{-10}$ & $-2.810 \cdot 10^{-14}$ & 0.09 \\
\hline 9 & -11.273 & $1.283 \cdot 10^{-2}$ & $-3.879 \cdot 10^{-6}$ & $5.534 \cdot 10^{-10}$ & $-3.016 \cdot 10^{-14}$ & 0.09 \\
\hline \hline
\end{tabular}

where

$$
\begin{aligned}
\log _{10} Q\left(T_{e}\right) & =-19.9171+0.0267 T_{e}-3.9960 \times 10^{-5} T_{e}{ }^{2} \\
+ & 3.5187 \times 10^{-8} T_{e}{ }^{3}-1.9228 \times 10^{-11} T_{e}{ }^{4} \\
+ & 6.6864 \times 10^{-22} T_{e}{ }^{7}-1.5346 \times 10^{-26} T_{e}{ }^{8} \\
& +5.0148 \times 10^{-31} T_{e}{ }^{9} .
\end{aligned}
$$

O fine structure [61]: 


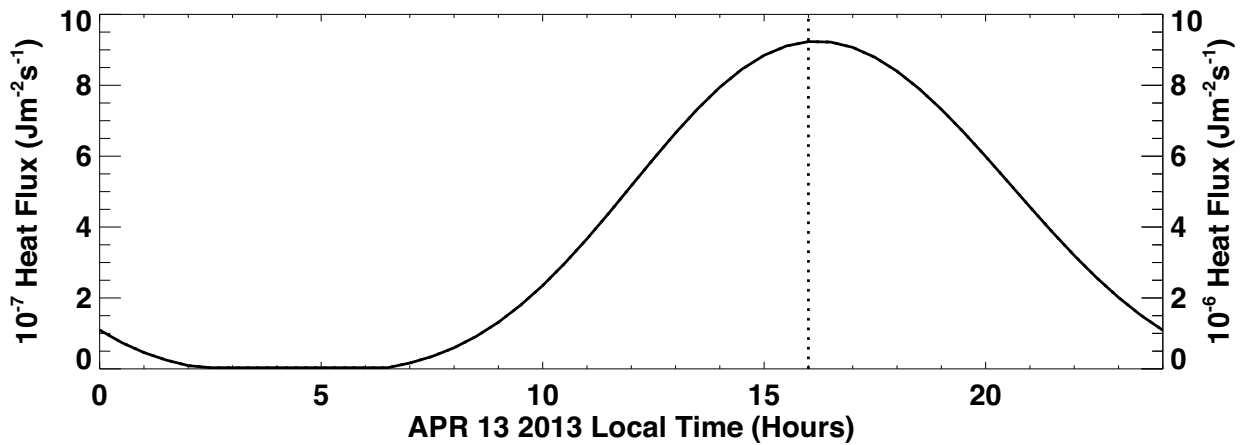

Figure 7. The electron heat flux specified on the topside of the ionosphere at Millstone ISR from 00 to 24 LT on April 13th, 2013. The left (right) Y-axis shows the magnitude of the small (large) heat flux.The dashed line marks 16 LT.

$$
\begin{gathered}
L_{e}(O)_{\text {fine }}=1 \times 10^{-12} n_{e} n(O) D^{-1}\left(S_{10}\left(1-e^{\left(98.9\left(T_{e}^{-1}-T_{n}^{-1}\right)\right)}\right)\right. \\
+S_{20}\left(1-e^{\left(326.6\left(T_{e}^{-1}-T_{n}^{-1}\right)\right)}\right) \\
\left.\quad+S_{21}\left(1-e^{\left(227.7\left(T_{e}^{-} 1-T_{n}^{-1}\right)\right)}\right)\right),
\end{gathered}
$$

where

$$
\begin{gathered}
D=5+e^{\left(-326.6 T_{n}^{-1}\right)}+3 e^{\left(-227.7 T_{n}^{-1}\right)}, \\
S_{21}=1.863 \cdot 10^{-11}, \\
S_{20}=1.191 \cdot 10^{-11}, \\
S_{10}=8.249 \cdot 10^{-16} T_{e}^{0.6} e^{\left(-227.7 T_{n}^{-1}\right)} .
\end{gathered}
$$

$\mathbf{O}\left({ }^{1} \mathbf{D}\right)$ excitation [62]:

$$
L_{e}\left(O\left({ }^{1} D\right)\right)=1.57 \times 10^{-24} n_{e} n(O) e^{\left(d \frac{T_{e}-3000}{3000 T_{e}}\right)}\left(e^{\left(-22713 \frac{T_{e}-T_{n}}{T_{e} T_{n}}\right)}-1\right)
$$

461

where

$$
d=2.4 \times 10^{4}+0.3\left(T_{e}-1500\right)-1.947 \times 10^{-5}\left(T_{e}-1500\right)\left(T_{e}-4000\right)
$$




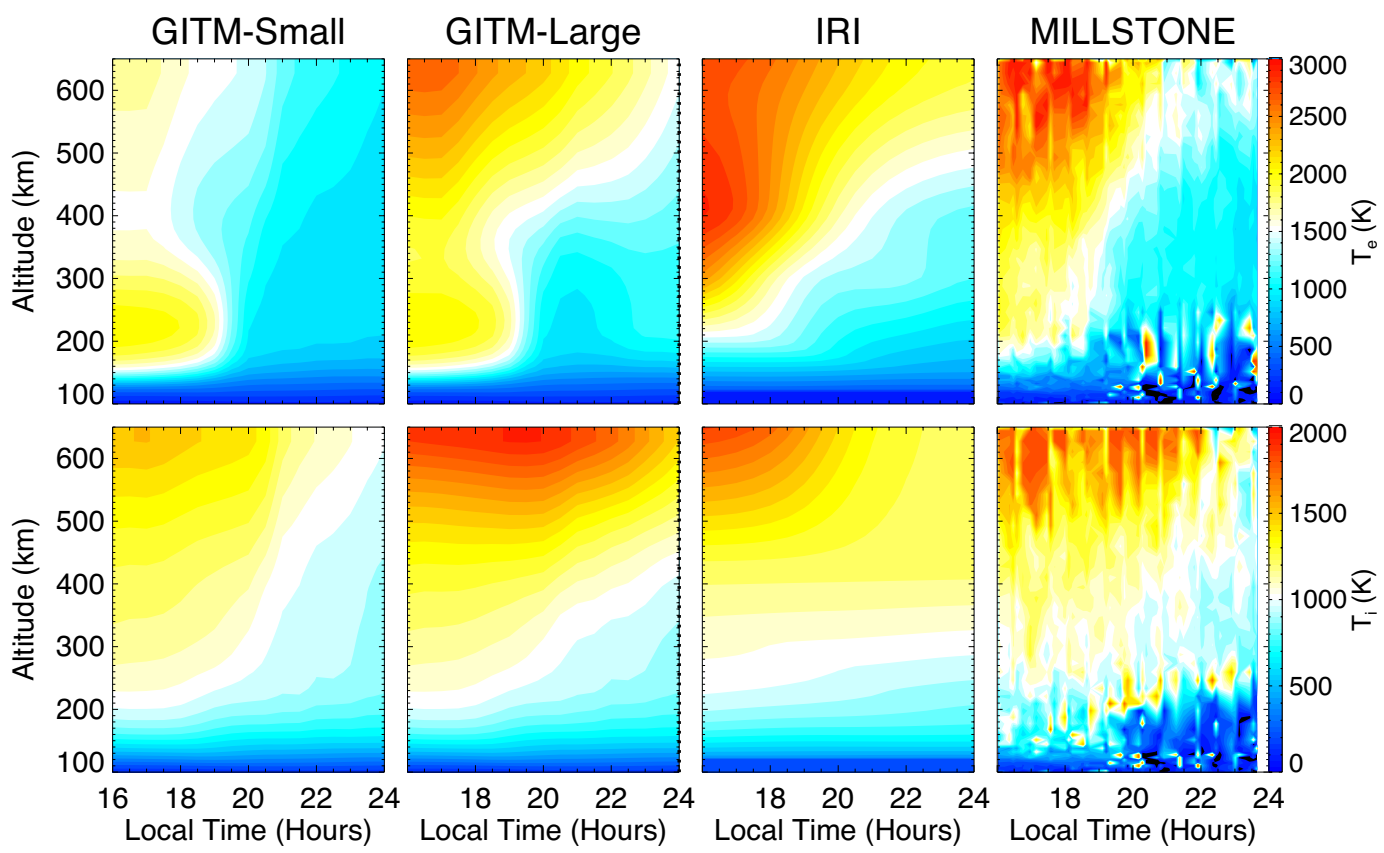

Figure 8. Comparisons of temporal variations in the electron temperature (top) and ion temperature (bottom) between the simulations (left three columns) and the Millstone observation (right colunm) from $100 \mathrm{~km}$ to $650 \mathrm{~km}$ from 16 to 24 LT on April 13th, 2013. The leftmost column shows $T_{e}$ and $T_{i}$ of the simulation driven by the small heat flux, while the second column from the left shows $T_{e}$ and $T_{i}$ of the simulation driven by the large heat flux. The third column column for the left shows results from IRI.

\section{Appendix B. Source Terms in the Calculation of the Ion Temperature} densities:

$$
\lambda_{i}=\frac{\sum_{t} n_{t} \lambda_{t}}{\sum_{t} n_{t}},
$$

where $n_{t}$ and $\lambda_{t}$ are the number density and the thermal conductivity of ion species $t$. The numerical expression for $\lambda_{t}$ is given by [2]:

$$
\lambda_{t}=3.1 \times 10^{6} \frac{T_{t}^{5 / 2}}{M_{t}^{1 / 2} Z_{t}^{4}}\left(1+1.75 \sum_{j \neq t} \frac{Z_{j}^{2}}{Z_{t}^{2}} \frac{n_{j}}{n_{t}}\left(\frac{M_{j}}{M_{t}+M_{j}}\right)^{1 / 2} \times \frac{3 M_{t}^{2}+1.6 M_{t} M_{j}+1.3 M_{j}^{2}}{\left(M_{t}+M_{j}\right)^{2}}\right)^{-1},
$$

where the unit is $\mathrm{eV} \mathrm{m}^{-1} \mathrm{~s}^{-1} \mathrm{~K}^{-1}$, the subscripts $t$ and $j$ refer to different ion species. Note that this expression is obtained for a fully ionized plasma in which ion-ion collisions are dominant. This expression may not apply well to the ionosphere at low altitudes, but is used anyways.

The collision frequencies for resonant ion-neutral interactions are given in Table Appendix B [63, 2]. The nonresonant collision frequencies are given by Schunk and Nagy [2]: 

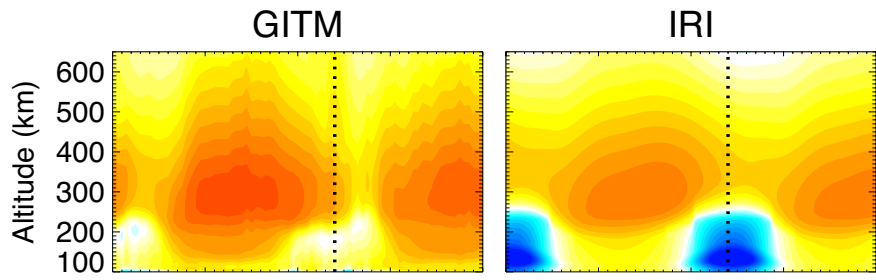

SONDRESTROM
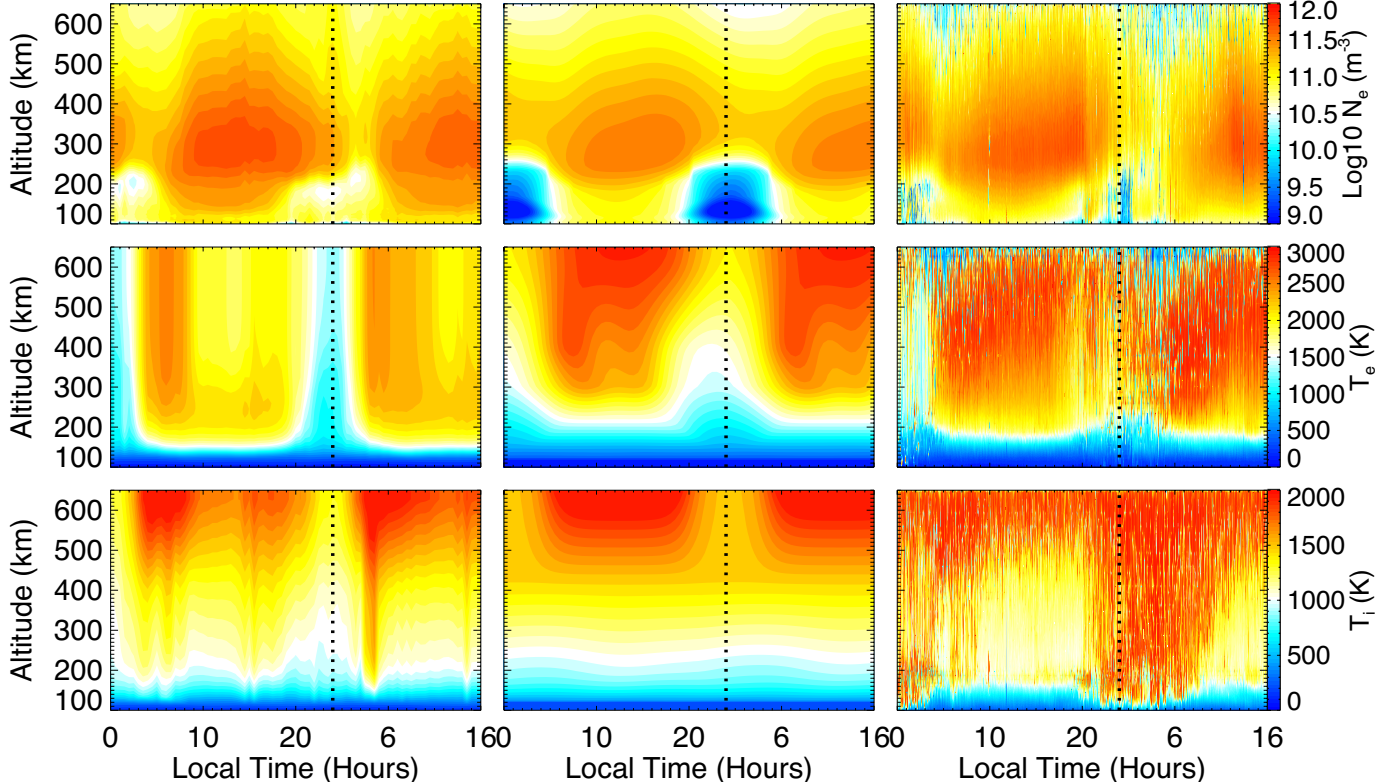

Figure 9. Comparisons of temporal variations in the electron density (top), electron temperature (mid) and ion temperature (bottom) between GITM (left column), IRI (center column) and observation by the Sondrestrom ISR (right column) from $100 \mathrm{~km}$ to $650 \mathrm{~km}$ on April 13 th and 14 th, 2013.

Table B.6. Collision frequencies for resonant ion-neutral interactions. $T_{r}=\left(T_{i}+T_{n}\right) / 2$ and densities are in $\mathrm{m}^{-3}$.

\begin{tabular}{|l|c|r|}
\hline \hline Species & $T_{r}, K$ & $v_{i n}, s^{-1}$ \\
\hline$N^{+}, N$ & $>275$ & $3.83 \times 10^{-17} n(N) T_{r}{ }^{1 / 2}\left(1-0.063 \log _{10} T_{r}\right)^{2}$ \\
\hline$O^{+}, O$ & $>235$ & $3.36 \times 10^{-17} n(O) T_{r}^{1 / 2}\left(1-0.064 \log _{10} T_{r}\right)^{2}$ \\
\hline$N_{2}{ }^{+}, N_{2}$ & $>170$ & $5.14 \times 10^{-17} n\left(N_{2}\right) T_{r}{ }^{1 / 2}\left(1-0.069 \log _{10} T_{r}\right)^{2}$ \\
\hline$O_{2}{ }^{+}, O_{2}$ & $>800$ & $2.59 \times 10^{-17} n\left(N_{2}\right) T_{r}^{1 / 2}\left(1-0.073 \log _{10} T_{r}\right)^{2}$ \\
\hline \hline
\end{tabular}

$$
v\left(O^{+}, O_{2}\right)=6.64 \times 10^{4} n\left(O_{2}\right),
$$

$$
v\left(O^{+}, N_{2}\right)=6.82 \times 10^{4} n\left(N_{2}\right),
$$

$$
v\left(\mathrm{O}_{2}{ }^{+}, N_{2}\right)=4.13 \times 10^{4} n\left(N_{2}\right),
$$

$$
v\left(\mathrm{O}_{2}{ }^{+}, O\right)=2.31 \times 10^{4} n(O),
$$

472 where the neutral densities are in $\mathrm{m}^{-3}$. 

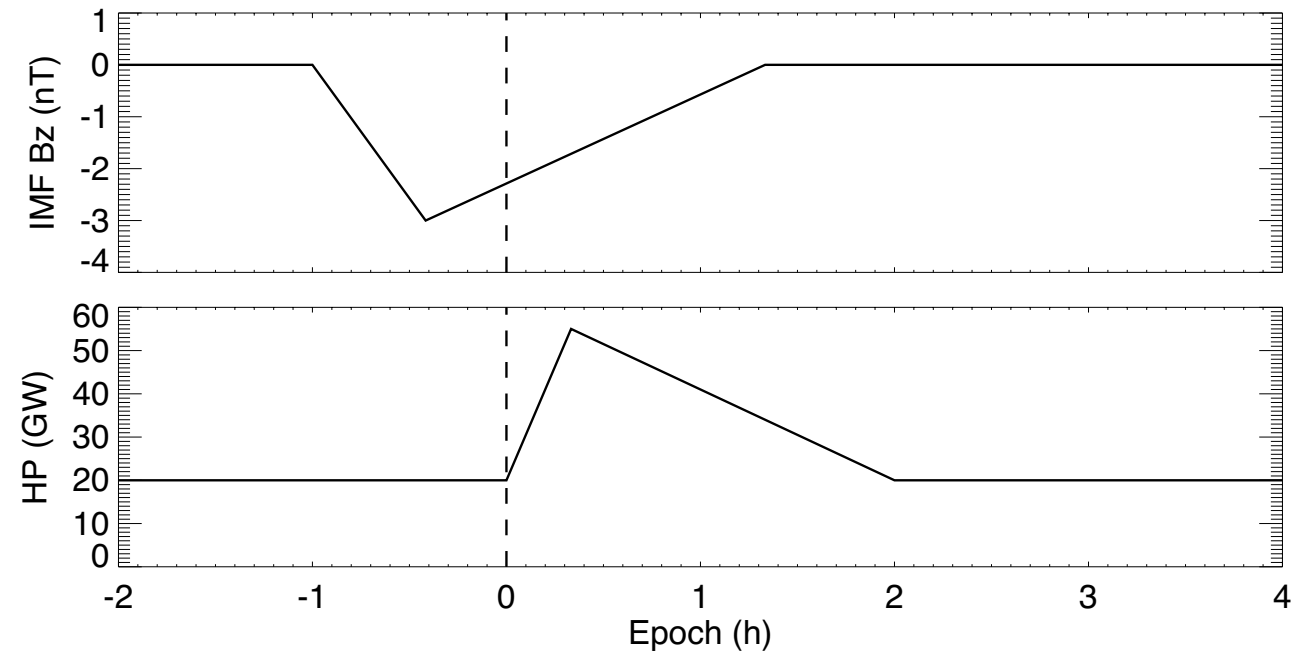

Figure 10. Temporal variations of IMF $B_{Z}$ and hemispheric power (HP) during an idealized substorm. The onset occurred at 0 epoch time, as indicated as the vertical dashed line.

\section{Appendix C. Charge Exchange and Recombination Reaction Rates}

The charge exchange rates $\kappa_{1}, \kappa_{2}, \kappa_{3}$ are given by [64, 65]:

$$
\kappa_{1}=1.533 \times 10^{-12}-5.92 \times 10^{-13}(T / 300)+8.6 \times 10^{-14}(T / 300)^{2}
$$

474 for $350 K \leq T \leq 1700 \mathrm{~K}$;

$$
\kappa_{1}=2.73 \times 10^{-12}-1.155 \times 10^{-12}(T / 300)+1.483 \times 10^{-13}(T / 300)^{2}
$$
for $1700 \mathrm{~K}<T<6000 \mathrm{~K}$.

$$
\kappa_{2}=2.82 \times 10^{-11}-7.74 \times 10^{-12}(T / 300)+1.073 \times 10^{-12}(T / 300)^{2}-5.17 \times 10^{-14}(T / 300)^{3}+9.65 \times 10^{-16}(T / 300)^{4}
$$

${ }_{476}$ for $350 \mathrm{~K} \leq T \leq 6000 \mathrm{~K}$.

$$
\kappa_{3}=8.36 \times 10^{-13}-2.02 \times 10^{-13}(T / 300)+6.95 \times 10^{-14}(T / 300)^{2}
$$



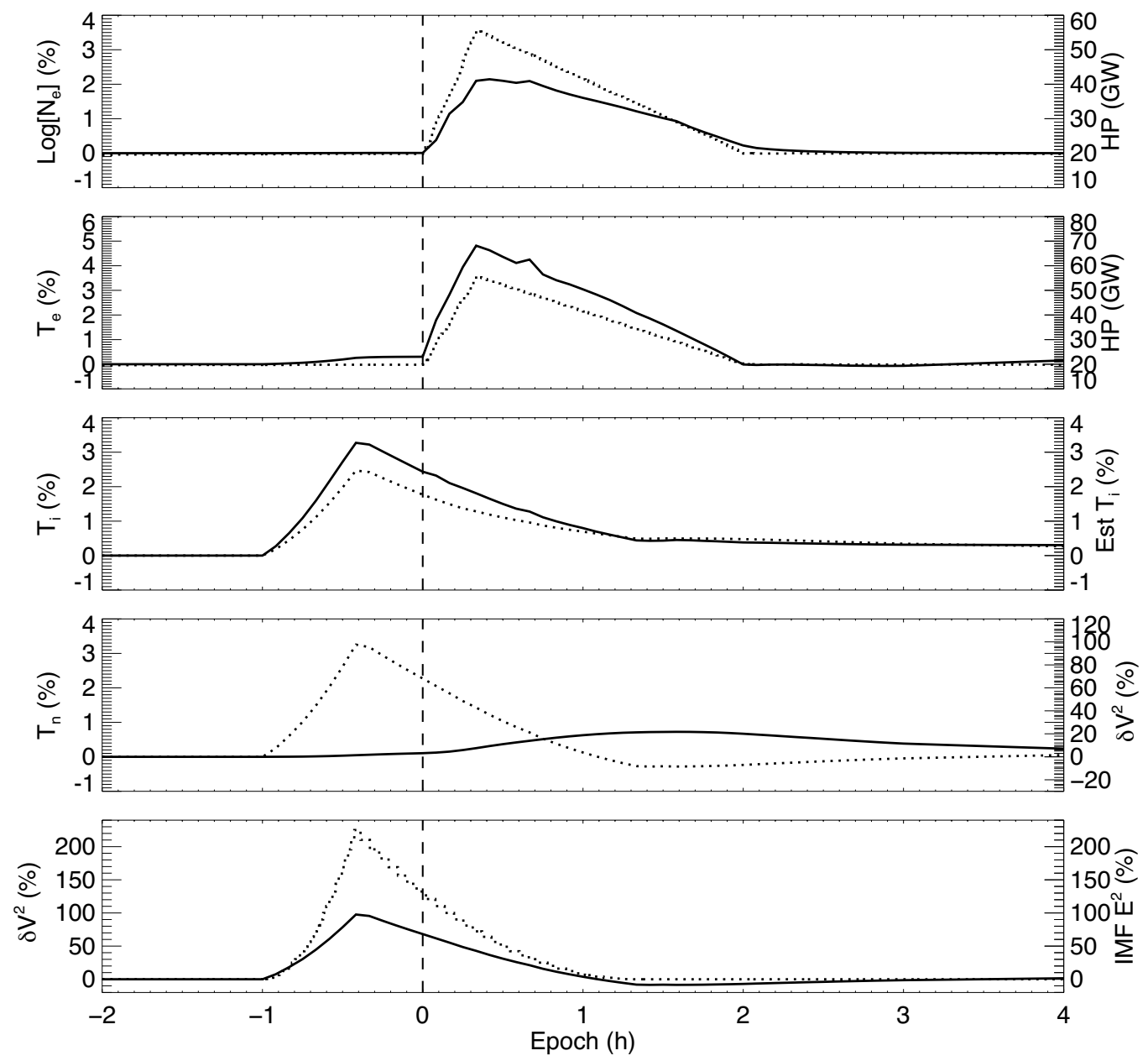

Figure 11. Solid lines show from top to bottom temporal variations of percentage difference in $N_{e}, T_{e}, T_{i}, T_{n}$ and square of velocity difference between ions and neutrals $\left(\delta V^{2}\right)$; dotted lines show from top to bottom HP, HP, the percentage difference in estimated $T_{i}, \delta V^{2}$ and square of IMF driven electric field. The ionospheric and thermospheric parameters are geometrically averaged over the north polar cap (above $40^{\circ}$ Latitude) at $140 \mathrm{~km}$.

$$
\kappa_{3}=5.33 \times 10^{-13}-1.64 \times 10^{-14}(T / 300)+4.72 \times 10^{-14}(T / 300)^{2}-7.05 \times 10^{-16}(T / 300)^{3}
$$

for $1500 \mathrm{~K}<T<6000 \mathrm{~K}$. In Eqn(C.1)-(C.5), $T$ is the effective temperature, which is given by:

$$
T=T\left(O^{+}\right)+\frac{m\left(O^{+}\right)}{m\left(O^{+}\right)+m_{r}} \frac{m_{r}-m_{b}}{3 \kappa} u_{\perp}^{2}\left(O^{+}\right),
$$

where

$$
m_{b}=\frac{\sum_{m} \frac{m_{n} v\left(O^{+}, n\right)}{m\left(O^{+}\right)+m_{n}}}{\sum_{m} \frac{v\left(O^{+}, n\right)}{m\left(O^{+}\right)+m_{n}}},
$$




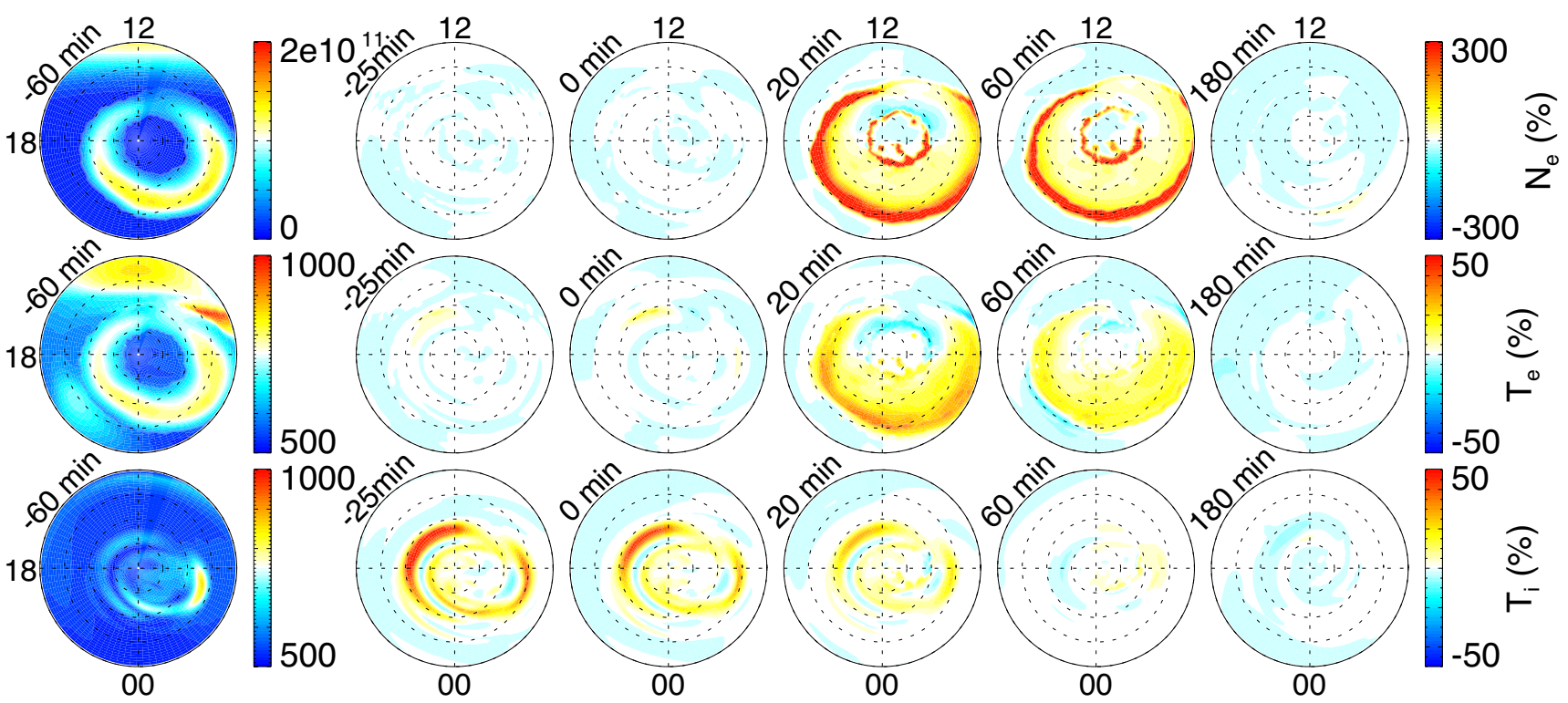

Figure 12. From top to bottom: spatial variations of $N_{e}, T_{e}$ and $T_{i}$ at $140-\mathrm{km}$ altitude at 6 epoch times during the substorm. The leftmost column shows the background ionosphere one hour before the substorm onset. Columns 2-6 show the percentage difference between the substorm simulation and the background simulation at $-20 \mathrm{~min}, 0 \mathrm{~min}, 20 \mathrm{~min}, 60 \mathrm{~min}$ and $180 \mathrm{~min}$ epoch times, respectively.

$m_{r}$ is the reactant mass of $\mathrm{N}_{2}, \mathrm{O}_{2}$ or $\mathrm{NO}$, and $T\left(\mathrm{O}^{+}\right)$is the $\mathrm{O}^{+}$temperature. Here, the transport effect was ignored, thus the effective temperature $T$ was reduced to the $O^{+}$temperature (equal the the bulk ion temperature in GITM).

The dissociative recombination rate $\left(\mathrm{in}^{-3}\right)$ of $\mathrm{NO}^{+}$is given by:

$$
\kappa_{4}=4.0 \times 10^{-13}\left(\frac{300}{T e}\right)^{0.5}
$$

The dissociative recombination rate (in $\mathrm{m}^{-3}$ ) of $O_{2}^{+}$is given by:

$$
\kappa_{5}=2.4 \times 10^{-13}\left(\frac{300}{T e}\right)^{0.7}
$$

The radiative recombination rate $\left(\mathrm{in}^{-3}\right.$ ) of $O^{+}$is given by:

$$
\kappa_{4}=3.7 \times 10^{-18}\left(\frac{300}{T e}\right)^{0.7}
$$

\section{Acknowledgement}

This work was partially supported by NASA Grant NNX09AJ59G and NSF grants AGS1242787 and AGS1138938. GITM utilized the ACE magnetometer and SWEPAM measurements as well. The link to the ACE data deposi- 

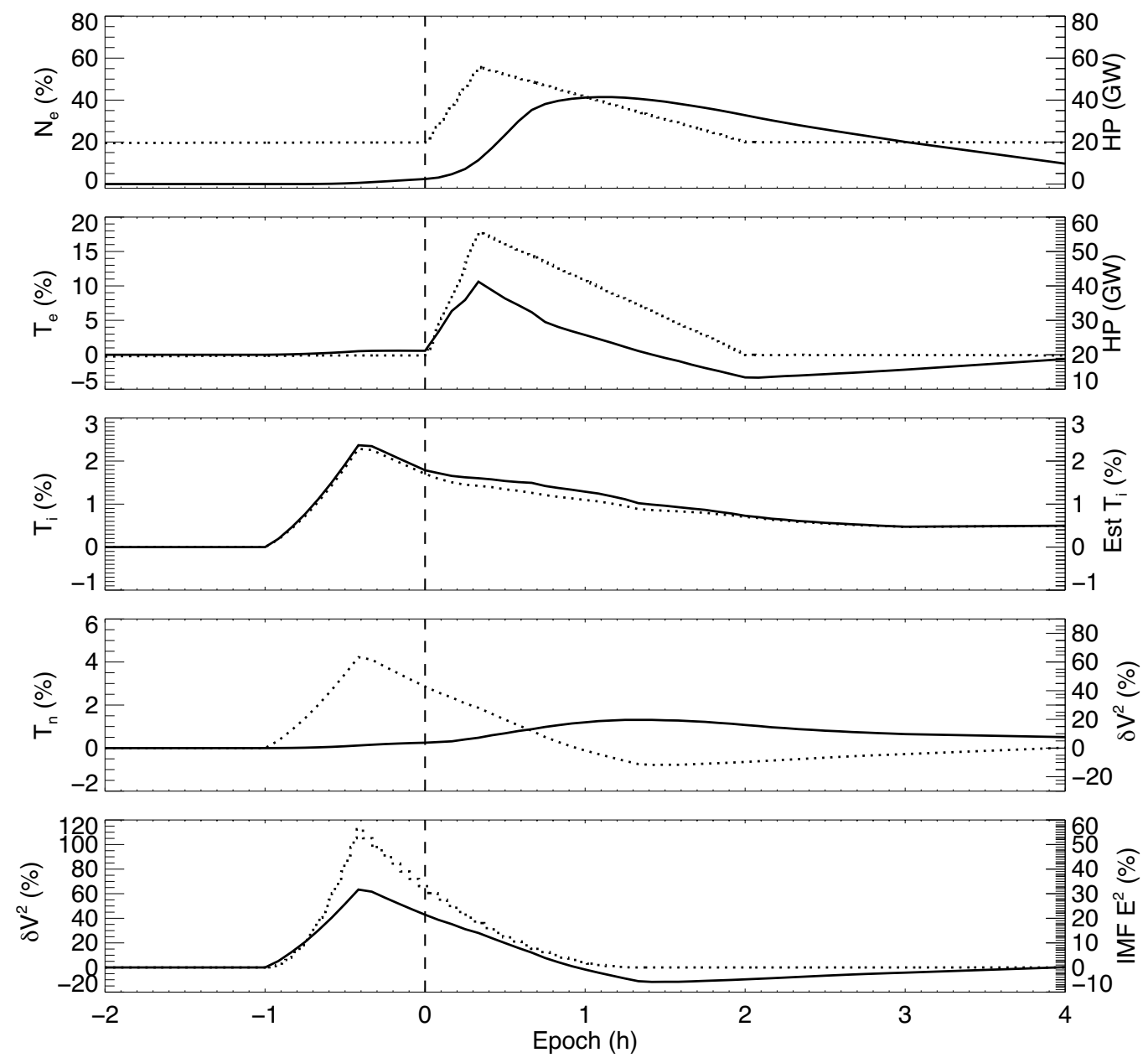

Figure 13. Same as Figure 11 but at $300 \mathrm{~km}$.

tory is http://www.srl.caltech.edu/ACE/ASC/level2/index.html, and the data are also available at http: //cdaweb.gsfc.nasa.gov/. The National Oceanic and Atmospheric Administration (NOAA) provided hemispheric power index data (http://www.swpc.noaa.gov/ftpmenu/lists/hpi.html) to drive the high-latitude auroral precipitation for this event. The ionospheric data measured by the Arecibo, Jicarmaca, Millstone and Sondrestrom incoherent scatter radars are available at http: //cedar.openmadrigal .org/. We would like to acknowledge high-performance computing support from Yellowstone (ark:/90890) provided by NCAR's Computational and Information Systems Laboratory, sponsored by the National Science Foundation. We would also like to thank NASA's supercomputers, Pleiades (http://www.nas.nasa.gov/hecc/resources/pleiades.html) for conducting the simulations in this study. The GITM simulation data for this case are available upon request from the authors. 


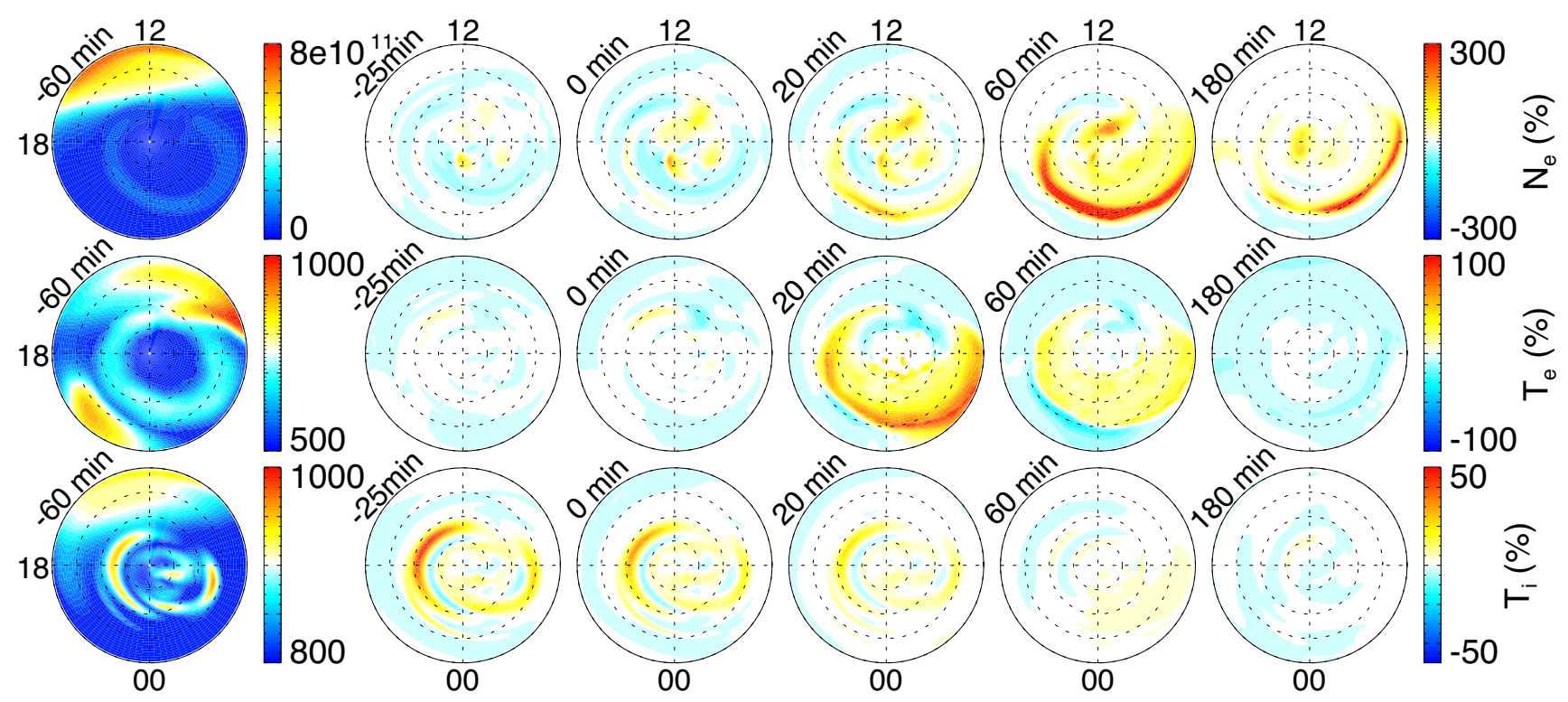

Figure 14. Same as Figure 12 but at $300 \mathrm{~km}$.

\section{References}

[1] L. B. N. Clausen, S. E. Milan, A. Grocott, Thermospheric density perturbations in response to substorms, jgr 119 (2014) $4441-4445$.

[2] R. Schunk, A. Nagy, Ionospheres: Physics, Plasma Physics, and Chemistry, Cambridge University Press, 2009.

[3] J. P. MaClure, Thermosphereic Temperature Variations Inferred from Incoherent Scatter Observations, J. Geophys. Res. 76 (1971) 3106.

[4] K. I. Oyama, S. Watanabe, Y. Su, T. Takahashi, K. Hirao, Season, local time, and longitude variations of electron temperature at the height of $\sim 600 \mathrm{~km}$ in the low latitude region, Adv. Space Res. 18 (1996) (6)269.

[5] S. Fukao, W. L. Oliver, Y. Onishi, T. Takami, t. Sato, T. Tsuda, M. Yamamoto, S. Kato, F-region seasonal behavior as measured by the MU radar, J. Atmos. Terr. Phys. 53 (1991) 599.

[6] A. Dalgarno, M. G. McElroy, R. J. Moffett, Electron Temperatures in the Ionosphere, Planet. Space Sci. 11 (1963) $463-484$.

[7] A. V. D. Rosa, The theoretical time-dependent thermal behavior of the ionospheric electron gas, J. Geophys. Res. 71 (1966) 4107.

[8] Y. Otsuka, S. Kawamura, N. Balan, S. Fukao, G. J. Bailey, Plasma temperature variations in the ionosphere over the middle and upper atmosphere radar, J. Geophys. Res. 103 (1998) 20705.

[9] J. Lei, R. G. Roble, W. Wang, B. A. Emery, S.-R. Zhang, Electron temperature climatology at Millstone Hill and Arecibo, J. Geophys. Res. 112 (2007) A02302.

[10] V. Truhlik, L. Triskova, D. Bilitza, K. Podolska, Variations of daytime and nighttime electron temperature and heat flux in the upper ionosphere, topside ionosphere and lower plasmasphere for low and high solar activity, Journal of Atmospheric and Solar-Terrestrial Physics. 71 (2009) 17-18.

[11] W. Wang, A. G. Burns, T. L. Killeen, A numerical study of the response of ionospheric electron temperautre to geomagnetic activity, Geophys. Res. Lett. 111 (2006) A11301

[12] R. W. Schunk, J. J. Sojka, M. D. Bowline, Theoretical Study of the Electron Temperature in the, High-Latitude Ionosphere for Solar Maximum and Winter Conditions, J. Geophys. Res. 91 (1986) 12041-12054. 
[13] M. David, R. W. Schunk, J. J. Sojka, The effect of downward electron heat flow and electron cooling processes in the high-latitude ionosphere, Journal of Atmospheric and Solar-Terrestrial Physics. 73 (2011) 1029-1048.

[14] B.-C. Zhang, Y. Kamide, Response of electron temperature to field-alighed current, J. Geophys. Res. 108 (2003) 1169.

[15] P. M. Banks, Ion Temperature in the Upper Atmosphere, J. Geophys. Res. 72 (1967) 3365-3385.

[16] R. W. Schunk, J. J. Sojka, Ion Temperature Variations in the Daytime High-latitude F Region, J. Geophys. Res. 87 (1962) 5169.

[17] J. P. Thayer, J. Semeter, The convergence of magnetospheric energ flux in the polar atmosphere, Journal of Atmospheric and Solar-Terrestrial Physics. 66 (2004) 807-824.

[18] R. W. Schunk, A mathematical model of middle and high-latitude ionosphere, Pure and Applied Geophysics 127 (1988) $255-303$.

[19] J. J. Sojka, Global scale physical models of the F region ionosphere, Rev. Geophys. 27 (1989) 371-403.

[20] G. H. Millward, R. J. Moffett, W. Quegan, T. J. Fuller-Rowell, A Coupled Thermosphere-Ionosphere-Plasmasphere Model, in STEP: Handbook of Ionospheric Models, National Technical Information Service, 5285 Port Pryal Road, Springfield, VA $22161,1996$.

[21] J. D. Huba, G. Joyce, Sami2 is Another Model of the Ionosphere (SAMI2): A new low-latitude ionosphere model, J. Geophys. Res. 105 (2000) 23,035-23,053.

[22] H. A. Observatory, TIEGCM V 1.94 Model Description, National Center for Atmospheric Research, Boulder, CO, 2011.

[23] W. Baumjohann, R. Pelunen, H. Opgenoorth, E. Nielsen, Joint two-dimensional observations of ground magnetic and ionospheric electric fields associated with auroral zone currents: Current systems associated with local auroral break-ups, Planetary and Space Science 29 (4) (1981) 431-447.

[24] B. Inhester, W. Baumjohann, R. Greenwald, E. Nielsen, Joint two-dimensional observations of ground magnetic and ionospheric electric fields associated with auroral zone currents. III- Auroral zone currents during the passage of a westward travelling surge, Journal of GeophysicsZeitschrift fuer Geophysik 49 (3) (1981) 155-162.

[25] H. Opgenoorth, I. Hägström, P. Williams, G. Jones, Regions of strongly enhanced perpendicular electric fields adjacent to auroral arcs, Journal of Atmospheric and Terrestrial Physics 52 (6) (1990) 449-458.

[26] B. N. Maehlum, T. Hansen, A. Brekke, O. Holt, K. Folkestad, Preliminary results from a study of the F-region heating during an intense aurora observed by EISCAT, jatp 40.

[27] K. R. Svenes, B. N. Maehlum, J. Trøim, G. Holmgren, R. L. Arnoldy, U. P. Løvhaug, M. T. Rietveld, Combined rocket and ground observations of electron heating in the ionospheric F-layer, pss 40.

[28] K. R. Svenes, B. N. Maehlum, U. P. Løvhaug, M. T. Rietveld, C. Hall, An investigation of two different electron heating events in the ionospheric F-layer, Advances in Space Research 12.

[29] T. Yeoman, J. Davies, N. Wade, G. Provan, S. E. Milan, Combined CUTLASS, EISCAT and ESR observations of ionospheric plasma flows at the onset of an isolated substorm, in: Annales Geophysicae, vol. 18, Springer, 1073-1087, 2000.

[30] X. Liu, A. Ridley, A simulation study of the thermosphere mass density response to substorms using GITM, jgr 120 .

[31] A. Ridley, Y. Deng, G. Tòth, The global ionosphere-thermosphere model, Journal of Atmospheric and Solar-Terrestrial Physics. 68 (2006) 839.

[32] A. D. Richmond, Ionospheric Electrodynamics Using Magnetic Apex Coordinates, J. Geomagn. Geoelectr. 47.

[33] G. Vichare, A. J. Ridley, E. Yiğit, Quiet-time low latitude ionospheric electrodynamics in the non-hydrostatic Global IonosphereThermosphere Model, Journal of Atmospheric and Solar-Terrestrial Physics. 80 (2012) 161-172.

[34] D. R. Weimer, Improved ionospheric electrodynamic models and application to calculating Joule heating rates, J. Geophys. Res. 110.

[35] T. J. Fuller-Rowell, D. S. Evans, Height-Integrated Pedersen and Hall Conductivity Patterns Inferred From the TIROS-NOAA Satellite Data, J. Geophys. Res. 92. 
[36] R. W. Schunk, J. C. G. Walker, Transport properties of the ionospheric electron gas, Planet. Space Sci. 18 (1970) 1535-1550.

[37] C. G. Smithtro, S. C. Solomon, An imporved parameterization of thermal electron heating by photoelectrons, with application to an X17 flare, J. Geophys. Res. 113 (2008) A08307.

[38] W. E. Swartz, J. S. Nisbet, Revised calculations of F region ambient electron heating by photoelectrons, J. Geophys. Res. 77 (1972) 62596261.

[39] S. C. Solomon, P. B. Hays, V. J. Abreu, The auroral 6300 Ã emission: Observations and modeling, J. Geophys. Res. 93 (1988) $9867-9882$.

[40] S. C. Solomon, V. J. Abreu, The auroral 630-nm dayglow, J. Geophys. Res. 94 (1989) 6817-6824.

[41] D. Bilitza, D. Altadill, Y. Zhang, C. Mertens, V. Truhlik, P. Richards, L.-A. McKinnell, B. Reinisch, The International Reference Ionosphere 2012-a model of international collaboration, Journal of Space Weather and Space Climate 4 (2014) A07.

[42] K. K. Mahajan, Models of electron temperature in the ionospheric F-region using electron density height profiles, J. Atmos. Terr. Phys. 139 (1976) 637-639.

[43] D. Bilitza, Electron and ion temperature data for ionospheric modelling, Adv. Space Res. 11 (1991) $139-148$.

[44] R. W. Schunk, A. F. Nagy, Electron temperatures in the F region of the ionosphere: Theory and observations, Rev. Geophys. 16 (1978) 355-399.

[45] D. Rees, T. J. Fuller-Rowell, The Response of the Thermosphere and Ionosphere to Magnetospheric Forcing, The Royal Society 328.

[46] W. E. Gordon, Incoherent Scattering of Radio Waves by Free Electrons with Applications to Space Exploration by Radar, Proceedings of the IRE 48 (1958) 1824-1829.

[47] M. W. Liemohn, J. U. Kozyra, P. G. Richards, G. V. Khazanov, M. J. Buonsanto, V. K. Jordanova, Ring current heating of the thermal electrons at solar maximum, J. Geophys. Res. 105 (2000) 27767-27776.

[48] H. A. Bekerat, R. W. Schunk, L. Scherliess, Estimation of the high-latitude topside electron heat flux using DMSP plasma density measurements, Journal of Atmospheric and Solar-Terrestrial Physics. 69 (2007) 1029-1048.

[49] L. R. Liuzzo, A. J. Ridley, N. J. Perlongo, E. J. Mitchell, M. Conde, D. L. Hampton, W. A. Bristow, M. J. Nicolls, High-latitude ionospheric dirvers and their effects on wind patterns in the thermosphere, J. Geophys. Res. 120 (2015) 715735.

[50] C. Reigber, H. Lühr, P. Schwintzer, CHAMP mission status, Advances in Space Research 30.

[51] J. R. Kan, L. C. Lee, Energy Coupling Function and Solar Wind-magnetosphere Dynamo, Geophys. Res. Lett. 6 (1979) 577-580, doi: \bibinfo\{doi\}\{10.1029/GL006i007p00577\}.

[52] J. St-Maurice, R. Schunk, Ion velocity distributions in the high-latitude ionosphere, Reviews of Geophysics and Space Physics 17 (1) (1979) 99-133.

[53] T. I. Gombosi, Physics of the Space Environment, Cambridge University Press, 1998.

[54] J.-E. Wahlund, H. Opgenoorth, F. Forme, M. Persson, I. Häggström, J. Lilensten, Electron energization in the topside auroral ionosphere: on the importance of ion-acoustic turbulence, Journal of atmospheric and terrestrial physics 55 (4) (1993) 623-645.

[55] Y. Itikawa, Momentum-transfer cross sections for electron collisions with atoms and molecules, Atmoic Data and Nuclear Data Tables 14 (1972) $1-10$.

[56] Y. Itikawa, Momentum transfer cross sections for electron collisions with atoms and molecules, At. Data Nucl. Data Tables. 14 (1978) 1-10.

[57] R. W. Schunk, A. F. Nagy, Ionospheres of the terrestrial planets, Rev. Geophys. Space Phys. 18 (1980) 813-852.

[58] A. V. Pavlov, New electron energy trnsfer rates for vibrational excitations of $N_{2}$, Ann. Geophys. 16 (1998) 176-182.

[59] A. V. Pavlov, New electron energy trnsfer and cooling rates by excitation of $O_{2}$, Ann. Geophys. 16 (1999) $1007-1013$.

[60] D. B. Jones, L. Campbell, M. J. Bottema, M. J. Brunger, New electron-energy trnsfer rates for vibrational excitation of $\mathrm{O}_{2}$, New Journal of Physics 5 (2003) 1007-1013. 
[61] A. V. Pavlov, K. A. Berrington, Cooling rate of thermal electrons by electrons by electron impact excitation of fine structure levels of atomic oxygen, Ann. Geophys. 17 (1999) 919-924

[62] R. J. W. Henry, P. G. Burke, A. L. Sinfailam, Scattering of electrons by C, N, O, $N^{+}, O^{+}$, and $O^{++}$, Phys. Rev. 178 (1969) $218-225$.

[63] P. M. Banks, G. Kockarts, Aeronomy, Academic Press, 1973.

[64] M. McFarland, D. L. Albritton, E. E. F. F. C. Fehsenfeld, A. L. Schmeltekopf, lowdrift technique for ion mobility and ionmolecule reaction rate constant measurements. II. Positive ion reactions of $\mathrm{N}^{+}, \mathrm{O}^{+}$, and $\mathrm{H}_{2}^{+}$with $\mathrm{O}_{2}$ and $\mathrm{O}^{+}$with $\mathrm{N}_{2}$ from thermal to $\sim 2 \mathrm{eV}$, J. Chem. Phys. 59 (1973) 6620

[65] R. W. Schunk, W. J. Raitt, P. M. Bank, Effect of electric fields on the daytime high-latitude E and F regions, J. Geophys. Res. 80 (1975) 31213130. 

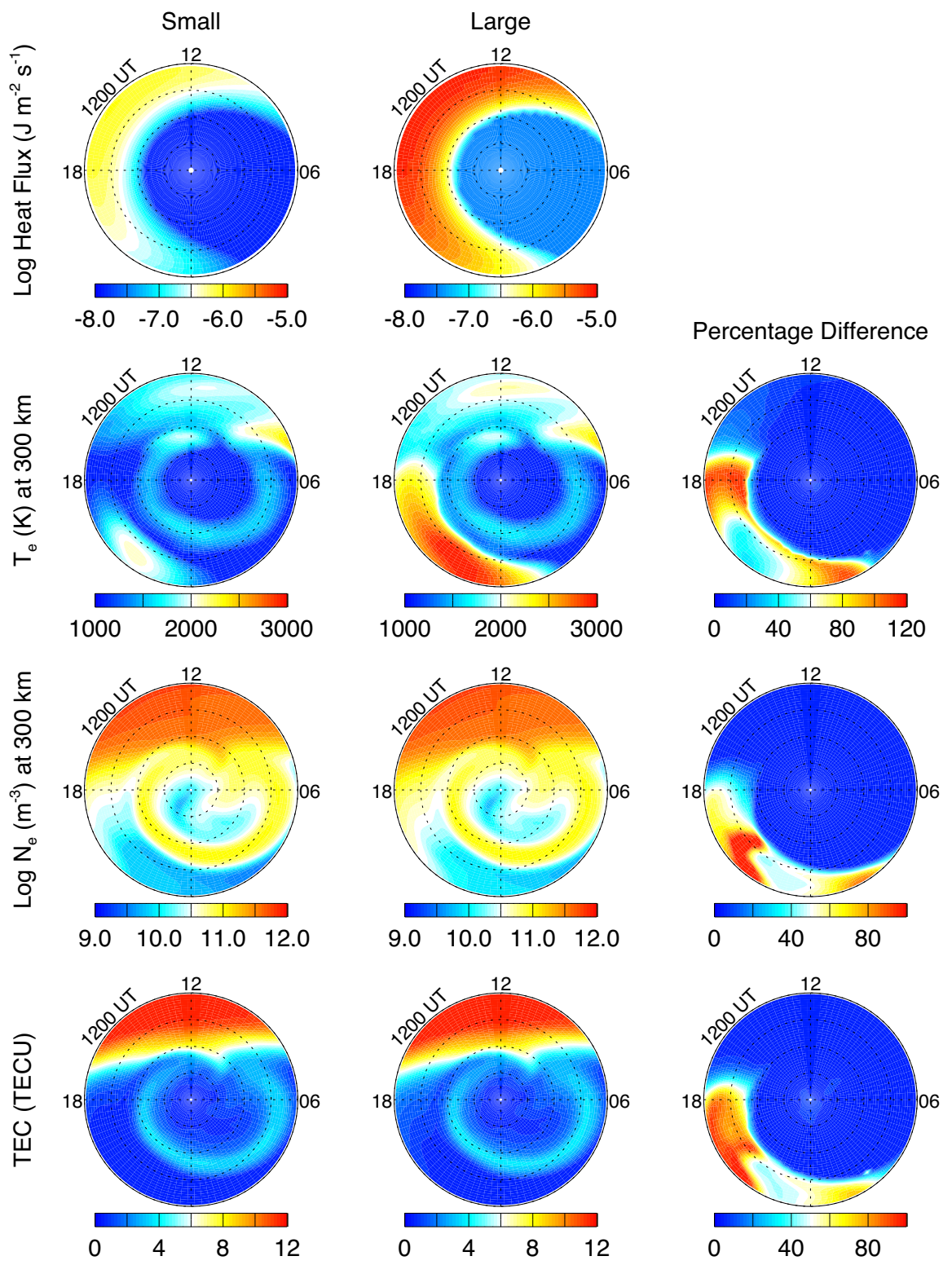

Figure 15. From top to bottom shows the electron heat flux into the ionosphere, $T_{e}$ and $N_{e}$ at an altitude of $300 \mathrm{~km}$ and the total electron content (TEC) at 12:00 UT . The first (second) column shows the simulation results driven by the small (large) heat flux, and the third column shows the percentage difference between the two. 


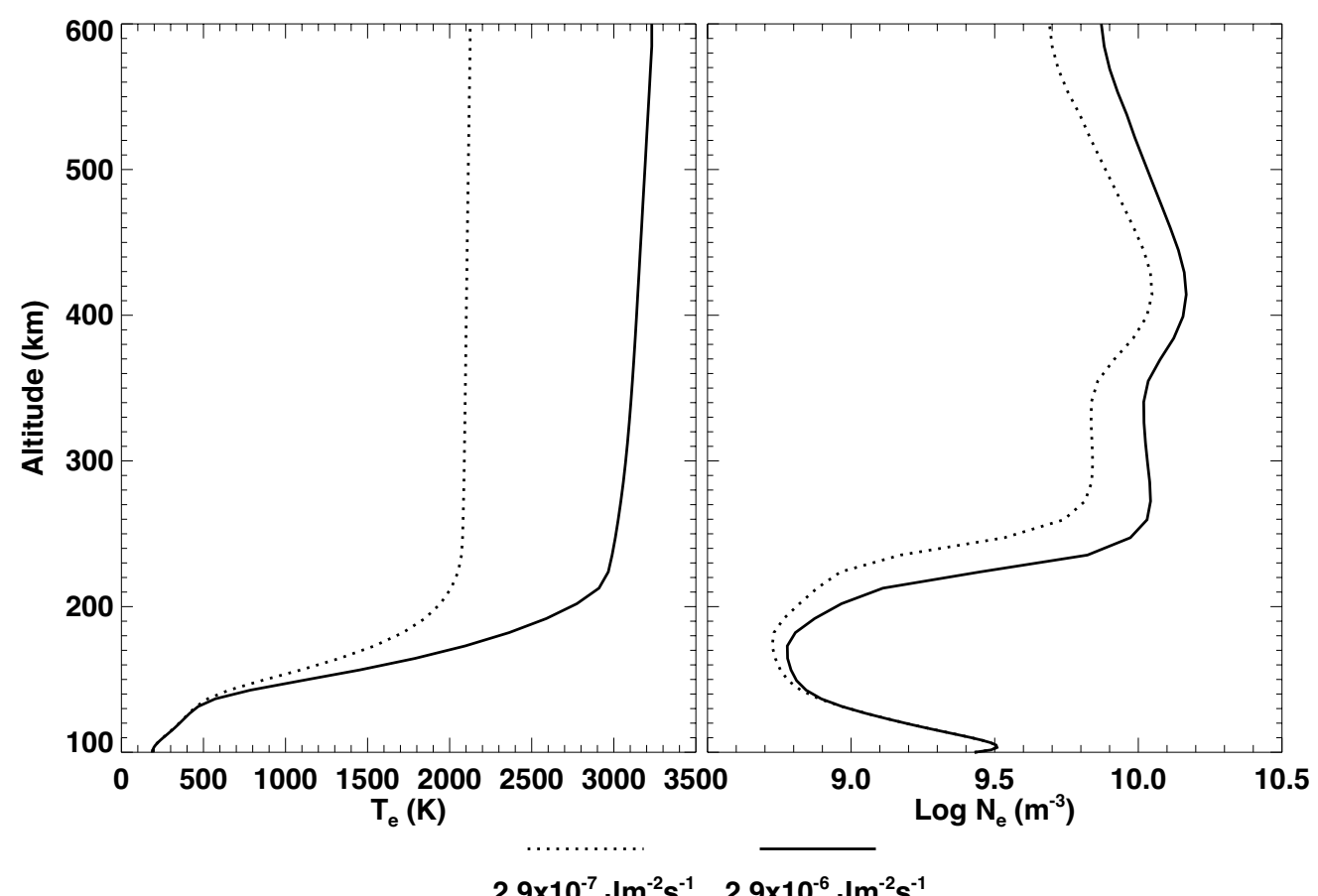

Figure 16. Altitudinal profiles of the electron temperature and electron density at $150^{\circ}$ Lon $55^{\circ}$ Lat at 12:00 UT (i.e., 22:00 LT). The dotted lines represent the top heat flux of $2.9 \times 10^{-7} \mathrm{Jm}^{-2} \mathrm{~s}^{-1}$, while the solid lines represent the top heat flux of $2.9 \times 10^{-6} \mathrm{Jm}^{-2} \mathrm{~s}^{-1}$. 


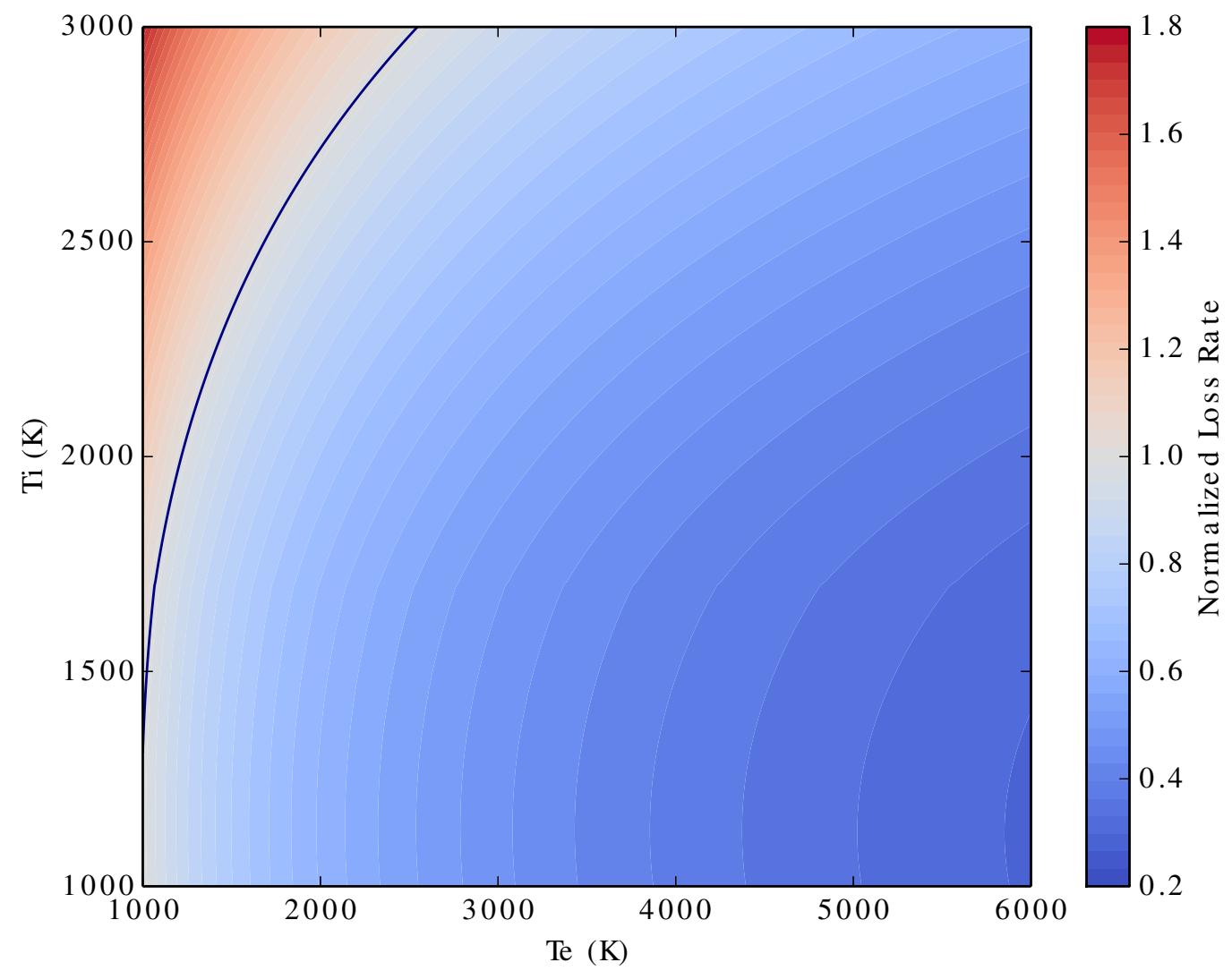

Figure 17. The electron density loss rate normalized to the value at $T e=1000 \mathrm{~K}, T_{i}=1000 \mathrm{~K}$. Level $=1.0$ is contoured by the solid line. 